\title{
Malacología Latinoamericana. Moluscos de agua dulce de Argentina
}

\author{
Alejandra Rumi, Diego E. Gutiérrez Gregoric, Verónica Núñez \& Gustavo A. Darrigran \\ División Zoología Invertebrados, Facultad de Ciencias Naturales y Museo, Universidad Nacional de La Plata, Paseo \\ del Bosque s/nº, 1900, La Plata, Buenos Aires, Argentina; alerumi@fcnym.unlp.edu.ar, dieguty@fcnym.unlp.edu.ar, \\ nmariaveronik@yahoo.com.ar, gdarrigran@malacologia.com.ar
}

Recibido 28-VI-2006. Corregido 14-II-2007. Aceptado 27-VII-2007.

\begin{abstract}
Latin American Malacology. Freshwater Mollusks from Argentina. A report and an updated list with comments on the species of freshwater molluscs of Argentina which covers an area of $2777815 \mathrm{~km}^{2}$ is presented. Distributions of Gastropoda and Bivalvia families, endemic, exotic, invasive as well as entities of sanitary importance are also studied and recommendations on their conservation are provided. Molluscs related to the Del Plata Basin have been thoroughly studied in comparison to others areas of the country. This fauna exhibits relatively the biggest specific richness and keeps its affinity with the fauna of other regions of the basin in areas of Paraguay and Brasil. The 4500 records of molluscs considered in this paper arise from the study of the collections of Museo Argentino de Ciencias Naturales "Bernardino Rivadavia", Buenos Aires; Museo de La Plata, La Plata and Fundación "Miguel Lillo", Tucumán. These institutions keep very important collections of molluscs in southern South America. Field information has recently been obtained and localities cited by other authors are also included in the data base. Until today, 166 species have been described, 101 belonging to 10 families of Gastropoda and 65 to 7 of Bivalvia. Families with highest specific richness are Lithoglyphidae (22) and Sphaeriidae (25), respectively. The number of endemic species (those present only in Argentina) by family is: Gastropoda: Ampullariidae (1), Cochliopidae (10), Lithoglyphidae (11), Thiariidae (3), Chilinidae (11), Lymnaeidae (2) and Physidae (2?); Bivalvia: Hyriidae (1?); Etheriidae (1?) and Sphaeriidae (10). Families with a distribution that comprise almost the whole country are: the Sphaeriidae and the gastropods Cochliopidae, Chilinidae and Lymnaeidae. Families Erodonidae and Solecurtidae (Bivalvia) were registered in mixohaline environments from Buenos Aires province. Gastropod families Thiaridae and Glacidorbiidae show a very restricted distribution. The rest of the families are present mainly in the center and north of the country. Species of sanitary interest are the propagators of: schistosomiasis-Biomphalaria peregrina, B. straminea y $B$. tenagophila, Planorbidae-, fasciolasis -Lymnaea viatrix and L. columnella, Lymnaeidae- and dermatitis -Chilina gibbosa and C. fluminea, Chilinidae. Invasive species are: Corbicula fluminea (Corbiculidae) and Limnoperna fortunei (Mytilidae). The construction of new areas for the protection and conservation of the high risk endemic species of freshwater molluscs is a priority. It is necessary to give special attention to the species of the patagonic mountain range and of the mesopotamic area of the Del Plata Basin. Rev. Biol. Trop. 56 (1): 77-111. Epub 2008 March 31.
\end{abstract}

Key words: freshwater molluscs, Gastropoda, Bivalvia, Argentina.

La República Argentina se sitúa en el extremo Sur de Sudamérica, entre los $21^{\circ}$ y $65^{\circ}$ $\mathrm{S}$ y $53^{\circ}$ y $73^{\circ} \mathrm{W}$, abarcando un área de 2777 $815 \mathrm{Km}^{2}$. Su población total es de 36260130 habitantes, con una tasa de crecimiento anual de $1.17 \%$ (Censo Nacional 2001). Alrededor del $45.5 \%$ de esa población está concentrada en las ciudades más importantes: unos $37.7 \%$ habitan en la Capital Federal y en el área metropolitana conocida como el Gran Buenos Aires. Presenta una gran variedad de hábitats: glaciares, desiertos áridos, altas montañas, estepas, pastizales y selvas. El clima varía desde subtropical en el Norte a templado frío en el Sur, con una variación térmica, en promedios anuales, que van desde $21.1 \mathrm{a}-5.6{ }^{\circ} \mathrm{C}$. 
El rango de humedad relativa es muy amplio, de extremadamente seco en el noroeste (con un promedio de $100 \mathrm{~mm}$ anuales de lluvias) a muy húmedo en algunas áreas de los bosques de la Patagonia en el extremo suroeste $(2500 \mathrm{~mm}$ de promedio anual de lluvias). La cordillera de los Andes conforma el límite oeste del país, con picos montañosos que alcanzan más de 7000 msnm. A pesar de que en la Argentina existen más de 250 áreas protegidas, que cubren algo más del $5 \%$ de la superficie del país, la actividad agroindustrial y el crecimiento desmedido de la población han causado la pérdida de más del $50 \%$ de sus bosques y selvas (WWF 1989, FVSA 2000).

Tres de los ambientes más biodiversos de América del Sur encuentran su límite de distribución austral dentro del territorio argentino: dos de ellos pertenecen a selvas, paranaense y yungas (Brown y Grau 1993), y el restante a los humedales del Chaco (Bucher y Chain 1998, Bertonatti y Corcuera 2000). Entre los taxa considerados para obtener tales valores de biodiversidad no fueron incluidos los moluscos.

Los ríos de Argentina se pueden separar en dos grandes grupos: los de la cuenca del Plata y los pertenecientes a la Región AndinoPatagónica, separadas ambas por una zona de transición con características intermedias (Fig. 1). De acuerdo con las estimaciones realizadas en WCMC (1998), la cuenca del Plata está considerada como una de las áreas de mayor aporte en biodiversidad de organismos de agua dulce. La presencia de numerosos saltos en sus cursos de agua, entre otras causas, posibilita la existencia de especies endémicas, especialmente en las áreas de la Mesopotamia Argentina.

Los primeros registros y descripciones de moluscos de la Argentina se realizaron en el siglo XIX (1834-37), cuando tuvo lugar la expedición conducida por Alcides d'Origny. En la segunda mitad de ese siglo y principios del XX, naturalistas europeos que vivían en la Argentina, como Ströbel y Dœring, describen otras tantas entidades, principalmente encontradas en la región central del país. En el siglo XX, Holmberg describe algunas especies, halladas especialmente en el Norte.
Los moluscos (Gastropoda y Bivalvia) relacionados a la cuenca del Plata, han sido los más estudiados en la Argentina, donde presentan relativamente la mayor riqueza específica, y retienen su afinidad con la de otras regiones de la cuenca, localizadas en áreas del Paraguay y Brasil.

El objetivo de este trabajo es presentar el estado de la situación y una lista actualizada sobre las especies de moluscos de agua dulce de la República Argentina, región política que como tal, desafortunadamente, careció de la contribución correspondiente en el marco de "Malacología Latinoamericana" (Barrientos y Monge-Nájera 2003). Además, se presenta la distribución a nivel de las familias de Gastropoda y Bivalvia y se realizan comentarios acerca de las entidades endémicas, exóticas, invasoras y de importancia sanitaria. Por último, se recomiendan algunas acciones para la conservación de la biodiversidad.

\section{MATERIALES Y MÉTODOS}

Los aproximadamente 4500 registros de moluscos considerados en este trabajo, fueron relevados de las colecciones del Museo Argentino de Ciencias Naturales "Bernardino Rivadavia", Buenos Aires (MACN); Museo de La Plata, La Plata (MLP) y Fundación "Miguel Lillo", Tucumán (FML). Estas instituciones albergan las colecciones de mayor envergadura y tradición en el desarrollo de la malacología en el Sur de Sudamérica. Al MLP fueron donadas las colecciones personales de M.I. Hylton Scott y A.A. Bonetto. Los registros también incluyen información de campo que se ha obtenido recientemente y las referenciadas por otros autores reconocidos: e.g. Carcelles (1941), Hylton Scott (1954, 1960, 1968), Castellanos (1959, 1981), Bonetto (1961, 1962, 1963, 1966, 1973), Parodiz (1965), Parodiz y Hennings (1965), Paraense (1974, 1985), Castellanos y Fernández (1976), Gaillard y Castellanos (1976), Olazarri (1977, 1980), Castellanos y Miquel (1980), Castellanos y Gaillard (1981), Castellanos y Landoni (1981), Cazzaniga 
(1981a), Fernández (1981a, b), Ituarte (1981, 1989, 1994, 1996, 2005), Bonetto et al. (1982), Tassara y Bechara (1983), Varela et al. (1983), López Armengol (1985), Rumi (1986, 1991), Bonetto y Tassara (1987), Darrigran (1991, 1995, 2004), Pastorino et al. (1993), Cuezzo y Drahg (1995), Miquel y Parent (1996), Rumi et al. (1997), Darrigran y López Armengol (1998), Peso y Quintana (1999).

Aquellas especies que solamente han sido descritas para la Argentina, incluyendo los ríos limítrofes, se consideran aquí endémicas. Para cada especie de la lista se describe un lote de colección como material de referencia. Las iniciales entre paréntesis corresponden a las de las provincias de origen del material (ver Fig. 1). Cuando la especie cuenta con muy pocos lotes depositados en las colecciones mencionadas, los números se adjuntan en la lista, en caso contrario ver Cuadro 1. Si la nomenclatura se ha indicado entre comillas (“"), significa que se mantiene la designación tradicional para estas especies en la Argentina, pero con profundas dudas acerca de su validez, cuestión que se aclara en los comentarios por familias correspondientes.

\section{Listado de especies}

\section{Clase Gastropoda}

Subclase Orthogastropoda Ponder \& Lindberg, 1996

Superorden Caenogastropoda Cox, 1960

Orden Architaenioglossa Haller, 1890

Superfam. Ampullarioidea Gray, 1824

Fam. Ampullariidae Gray, 1824

Gen. Asolene d'Orbigny, 1875

A. platae (Maton, 1809); MLP=361, Río Santiago (BA).

A. puelchella (Anton, 1839); MLP=360, Laguna Guadalupe (SF).

A. spixii (d'Orbigny, 1835); MLP=10932, Río Batel (CR).

Gen. Felipponea Dall, 1919

F. neritiniformis (Dall, 1919); MLP= 5338, La Cruz (CR) y 8822 al 8828.

F. elongata (Dall, 1921); MLP=6678, Federación (ER) y 8819 .

F. iheringi (Pilsbry, 1933); MLP= 8820, Federación (ER) y 8821.

Gen. Marisa Gray, 1824

M. planogyra Pilsbry, 1933; MLP=359, Laguna Guadalupe (SF).

Gen. Pomacea Perry, 1810

P. (Pomacea) canaliculata (Lamarck, 1822); MLP= 281, Silva (SF).

P. (P) insularum (d'Orbigny, 1835); MLP=341, Iguazú (MS) y 3360.

P. (P) scalaris (d'Orbigny, 1835); MLP=358, Río Paraná (CO).

Gen. Pomella Gray, 1847

P. (Pomella) americanista (Ihering, 1919); MACN= 8776, Iguazú (MS) (Cotipos).

P. (P.) megastoma (G. B. Sowerby I, 1825); MLP=1187, San Javier (MS).

Comentarios: Para la nomenclatura de estas especies se siguió a la revisión realizada por Cowie y Thiengo (2003). Pomacea insularum: posible sinónimo de Pomacea gigas (Spixi, 1827) (Castellanos y Fernández 1976, Cowie y Thiengo 2003). El Lote del MLP N 11359 corresponde al sintipo de P. canaliculata chaquensis. 
Orden Sorbeoconcha Ponder \& Lindberg, 1997

Superfam. Cerithioidea Fèrusac, 1822

Fam. Thiaridae Troschell, 1857

Gen. Aylacostoma Spix, 1827

A. chloroticum Hylton Scott, 1954; MLP= 10958 y 11596, Alto Paraná (CR) (Paratipo).

A. guaraniticum (Hylton Scott, 1951); MLP=11213, Alto Paraná (CR) (Paratipo); 388, 6712 y 9083.

A. stigmaticum Hylton Scott, 1954; MLP= 10963 al 10965, Alto Paraná (CR) (Paratipo).

Gen. Melanoides Oliver, 1804

M. tuberculatus (Müller, 1774); sin material de referencia en colecciones.

Comentarios: Se siguió el criterio de Castellanos (1981) -donde figura la lista sinonímica de la familia- y de Quintana y Mercado Lackzó (1997). No fueron tomados en cuenta Aylacostoma cingulatum Hylton Scott in schedule y un nuevo morfotipo (Quintana y Mercado Lackzó 1997) por no estar hasta el presente apropiadamente descritos.

Superfam Rissooidea Gray, 1847

Comentarios: Se siguieron los criterios de Wilke et al. (2001) para el ordenamiento de esta superfamilia, ya que se basaron en estudios morfoanatómicos, moleculares y filogenéticos.

Fam. Cochliopidae Tryon, 1866

Gen. Heleobia Stimpson, 1865

H. australis (d'Orbigny, 1835); MLP=4049, Golfo San Matías (RN) (Tipo).

H. castellanosae (Gaillard, 1974); MLP=4052, Palermo (Cap. Federal) (Sintipo).

H. conexa (Gaillard, 1974); MLP=4053, Mar Chiquita (BA) (Sintipo).

H. guaranitica (Doering, 1884); MLP= 4441, Laguna Los Matadores (SF); 9365 y 9366.

H. hatcheri (Pilsbry, 1911); MLP=373, Aguada del Overo (NQ).

H. isabelleana (d'Orbigny, 1835); MACN= 28791, Bahia Samborombón (BA).

H. kuesteri (Ströbel, 1874); FML= 5813, Uspallata (MZ) y 10727.

H. montana (Doering, 1884); MLP=9153, Aguas de Ramón (CB). MACN= 9543.

H. occidentalis (Doering, 1884); MLP=9097, Salinas del Timbó (TC).

H. parchappii (d'Orbigny, 1835); MLP=11746, Paso Otero (BA).

H. peiranoi (Weyrauch, 1963); FML= 41, Serranía del Timbó (TC) (Holotipo).

H. piscium (d'Orbigny, 1835); MLP=6760, Isla Martín García (BA).

H. rionegrina (Gaillard, 1974); MLP=4050, Arroyo Valcheta $(\mathrm{RN})$ (Sintipo). $\mathrm{MACN}=28638$.

H. sublineata (Pilsbry, 1911); sin material de referencia en colecciones.

H. tucumana (Gaillard, 1974); MLP= 4051, Arroyo El Tala (TC) (Serie Tipo).

H. vianai (Parodiz, 1960); MLP=387, Lago Brea (JU); 4460; 9224 y 9225. MACN=930 y 20836.

Comentarios: Según Davis et al. (1982), se incluye en el género Heleobia, a Littoridina australis y a las especies relacionadas de Brasil, Uruguay y Argentina. De acuerdo a Kabat y Hershler (1993), Strobeliella Cazzaniga, 1981 es sinonimo de Heleobia. Gaillard y Castellanos (1976) publicaron la lista sinonímica de esta familia. Cazzaniga (1981b) menciona a H. vianai como un posible sinonimo de H. occidentalis. Heleobia scotti (Pilsbry, 1911) no fue considerada en el listado por poseer información geográfica incompleta. Heleobia isabellana (Localidad tipo, Montevideo, Uruguay) no fue incluida en la lista de especies de Uruguay por Sacarabino (2004). 
Fam. Lithoglyphidae Troschel, 1857

Gen. Potamolithus Pilsbry, 1896

P. agapetus Pilsbry, 1911; MLP $=4650$, San Isidro (BA).

P. buschii (Frauenfeld, 1865); MLP= 4621, Isla Martín Garcia (BA).

P. callosus Pilsbry, 1925; MLP=4615, Federación (ER); 4632 y 9942. $\mathrm{MACN}=17335$.

P. catharinae Pilsbry, 1911; sin material de referencia en colecciones.

P. concordianus Parodiz, 1966; MACN=17334, Río Uruguay (ER).

P. conicus (Brot, 1867); MLP=4623, El Palmar (ER) y 4631. MACN=17334 al 17336.

P. dinochilus Pilsbry, 1896; MLP=4615, Federación (ER).

P. doeringi Pilsbry, 1911; MLP=4617, Arroyo Uruqui (MS); 4618; 4619; 4646 al 4648.

P. hidalgoi Pilsbry, 1896; MLP= 4615, Federación (ER) y 371 .

P. iheringi Pilsbry, 1896; MLP= 4615, Federación (ER); 4616; 4622 y 4635.

P. lapidum (d'Orbigny, 1835); MLP=371, San Javier (MS).

P. microthauma Pilsbry, 1896; MLP=371, San Javier (MS) y 9948. MACN=25793.

P. orbignyi Pilsbry, 1896; MLP= 4623, El Palmar (ER).

P. paranensis Pilsbry, 1911; MLP=4614, Paso de la Patria (CR).

P. peristomatus (d'Orbigny, 1835); MLP= 4618, Iguazú (MS).

P. petitianus d'Orbigny, 1840; MLP= 4623, El Palmar (ER); 4651 у 9941.

P. philipianus Pilsbry, 1911; MLP=4616, Monte Caseros (CR).

P. quadratus Pilsbry \& Ihering, 1911; MACN=17338, Concordia (ER).

P. rushii Pilsbry, 1896; MLP $=4615$, Federación (ER) y 371. MACN $=17344$.

P. simplex Pilsbry, 1911; MLP=4623, El Palmar (ER) y 4625. MACN=17111.

P. tricostatus (Brot, 1867); MLP= 4623, El Palmar (ER) y 4644. MACN=17334.

P. valchetensis Miquel, 1998; MACN=3411, Arroyo. Zoilo Chico (RN) (Holotipo).

Comentarios: Potamolithus jacuhyensis Pilsbry, 1899 fue mencionado por Parodiz (1965), pero en posteriores revisiones se la considera presente solamente en Brasil (López Armengol 1985). Asimismo, ningún lote fue hallado en las colecciones consultadas. López Armengol (1985) publica la lista sinonímica de la familia, luego completada por López Armengol y Darrigran (1998).

Superorden Heterobranchia Burmeister, 1837

Orden Allogastropoda Haszprunar, 1985

Superfam. Glacidorboidea Ponder, 1986

Fam. Glacidorbidae Ponder, 1986

Gen. Gondwanorbis Ponder, 1986

G. magallanicus (Meier-Brook \& Smith, 1976); MLP= 5503, Lago Yehuín (TF) y 5504.

Comentarios: Originalmente bajo la denominación de Glacidorbis magallanicus Meier-Brook y Smith, 1976. Según Bouchet y Rocroi (2005) esta familia pertenece a un grupo informal al que denominan "Lower Heterobranchia" (= Allogastropoda).

Orden Pulmonata Cuvier, 1814

Superfam. Chilinoidea Adams \& Adams, 1855

Fam. Chilinidae Dall, 1807

Gen. Chilina Gray, 1828

C. aurantia Marshall, 1924; MACN=23298, Junín de Los Andes (NQ).

C. dombeiana (Bruguiere, 1789); MLP=769, Lago Buenos Aires (SC).

C. fluminea (Maton, 1809); MLP=386, Río de la Plata (BA). 

C. fulgurata Pilsbry, 1911; MLP=2262, Lago Posadas (SC).
C. gallardoi Castellanos \& Gaillard, 1981; MLP=4096, San Javier (MS) (Paratipo).
C. gibbosa G. B. Sowerby I, 1841; MLP=698, Collon Curá (NQ).
C. guaraniana Castellanos \& Miquel, 1980; MACN= 568, Posadas (MS) (Tipo).
C. megastoma Hylton Scott, 1958; MLP=11068, Iguazú (MS) (Tipo).
C. mendozana Ströbel, 1874; MLP=7391, Mendoza (MZ); 8260 y 8261.
C. neuquenensis Marshall, 1933; MLP= 8262, Lago Correntoso (NQ). MACN=6403.
C. parchappii (d'Orbigny, 1835); MLP=764, Arroyo Valcheta (RN).
C. patagonica Sowerby II, 1874; MLP= 2903, Río Gallegos (SC).
C. perrieri Mabille, 1833; MLP= 4102, Piedra Buena (SC) y 29408.
C. portillensis Hidalgo, 1880; MLP= 2967, Ledesma (JU) y 2983.
C. rushii Pilsbry, 1911; MLP=8276, Gualeguaychú (ER); y 8277 al 8279.
C. strebeli Pilsbry, 1911; MLP=6725, Cañadón de las Vacas (SC). MACN= 3104 y 23215.

Comentarios: Castellanos y Gaillard (1981) publican la lista sinonímica de esta familia. El material de $C$. guaraniana citado como depositado en la colección del MLP por Castellanos y Miquel (1980), no figura en la base de datos de dicha colección. Los lotes No 9927 (Paratipo, Río Cochuna (TC)) y 9954 del MACN corresponden a $C$. fluminea tucumanensis. Actualmente las especies de esta familia están sujetas a revisión por el equipo de trabajo.

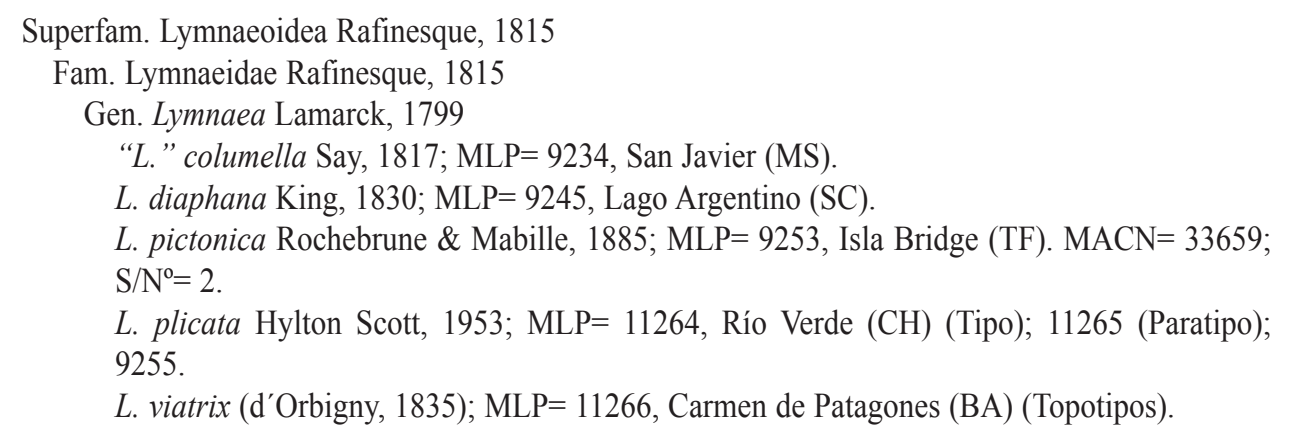

Comentarios: Castellanos y Landoni (1981) publican la lista sinonímica de esta familia. No existe acuerdo entre los especialistas respecto a la ubicación genérica de "L." columella: Paraense (1983) la denomina en aquel género, mientras que, para Hylton Scott (1953), Walter (1968), Castellanos y Landoni (1981) y Rudolph (1983) corresponde la denominación de Pseudosuccinea columella (Say, 1817).

Superfam. Planorboidea Rafinesque, 1815

Fam. Planorbidae Rafinesque, 1815

Gen. Antillorbis Harry \& Hubendick, 1963

A. nordestensis (Lucena, 1954); MLP=7015, Isla Martín Garcia (BA).

Gen. Acrorbis Odhner, 1937

A. petricola Odhner, 1937; MLP= 5089, Salto Encantado (MS).

Gen. Biomphalaria Preston, 1910

B. intermedia (Paraense \& Deslandes, 1962); sin material de referencia en colecciones.

B. occidentalis Paraense, 1981; MLP=7162, Puerto Bermejo Viejo (CO).

B. oligoza Paraense, 1974; MLP=11956, San Roque (CB).

B. orbignyi Paraense, 1975; MLP=2261, Paraná (ER); 6997; 7167; 7787; 7788.

B. peregrina (d'Orbigny, 1835); MLP=780, La Mansilla (CB). 
B. straminea (Dunker, 1848); MLP=7850, San José (MS).

B. tenagophila (d'Orbigny, 1835); MLP=350, Mercedes (CR).

Gen. Drepanotrema Fischer \& Crosse, 1880

D. anatinum (d'Orbigny, 1835); MLP=4441, Laguna Los Matadores (SF).

D. cimex (Moricand, 1839); MLP= 6757 al 6759, Isla Martín García (BA).

D. depressissimun (Moricand, 1839); MLP= 5324, Río Salado (TC).

D. heloicum (d'Orbigny, 1835); MLP= 8534, Las Rosas (BA); 8536; 8537. MACN= S/ $\mathrm{N}^{\circ}=1$

D. kermatoides (d'Orbigny, 1835); MLP=2136, Luján (BA).

D. lucidum (Pfeiffer, 1839); MLP=7004, Itacaruaré (MS).

Comentarios: Rumi (1991) publica la lista sinonímica de esta familia. Según Rumi (1986) para los saltos de Apipé, en la provincia de Corrientes, fue registrada la presencia de Acrorbis sp. Actualmente estos saltos han sido modificados por el lago de inundación de la represa de Yacyretá.

Fam. Ancylidae Rafinesque, 1815

Gen. Anisancylus Pilsbry, 1924

A. obliquus (Broderip \& G. B. Sowerby I, 1832); MLP=2154, Río Primero (CB).

Gen. Gundlachia Pfeiffer, 1849

G. ticaga (Marcus \& Marcus, 1962); sin material de referencia en colecciones.

Gen. Hebetancylus Pilsbry, 1913

H. moricandi (d'Orbigny, 1837); MLP=3610, Ibicuicito (ER).

Gen. Laevapex Walker, 1903

Laevapex sp.; sin material de referencia en colecciones.

Gen. Uncancylus Pilsbry, 1913

U. concentricus (d'Orbigny, 1835); MLP=2153, Arroyo Saldán (CB).

Comentarios: Para Ancylidae se siguió a Barbosa dos Santos (2003). Fernández (1981) publica la lista sinonímica de esta familia. Nosotros consideramos a los Ancylidae como una familia, pero Bouchet y Rocroi (2005) los incluye en la tribu Ancylini dentro de los Planorbidae.

Superfam. Physacea Harry \& Hubendick, 1964

Fam. Physidae Fitzinger, 1833

Gen. Physa Draparnaud, 1801

P. aspii Holmerg, 1909; MACN=1407, Laguna de los Murciélagos (FO).

P. loosi Holmerg, 1909; MACN=1404, Santa Lucia (SJ).

Gen. Physella Haldeman, 1843

"P. cubensis" (Pfeiffer, 1839); MACN= 32605, Punta Lara (BA); 34476.

"P." venustula (Gould, 1848); sin material de referencia en colecciones.

Gen. Stenophysa Martens, 1898

"S. marmorata" (Guilding, 1828); MLP=7193, Iguazú (MS).

Comentarios: Esta familia es aún objeto de importantes revisiones sistemáticas. Según la última revisión realizada por Taylor (2003), P. loosi y P. aspii estarían categorizadas como incertae sedis, ya que sólo fueron descritas y mencionadas por Holmberg. Taylor (2003) sitúa a Physa acuta Draparnaud, 1805, P. cubensis y $P$. venustula dentro del género Haitia Clench \& Aguayo, 1932, basándose en la ausencia de tejido glandular en la vaina del pene y considera que la especie presente en la Argentina es Haitia acuta o Haitia mexicana (Philippi, 1841). Sin embargo, Paraense y Pointier (2003) consideran a P. cubensis como sinónimo de P. acuta. Taylor 
(2003 y 2004) además señala que la distribución de "S. marmorata" no alcanzaría el territorio argentino, y que los especimenes de la Argentina podrían pertenecer a otra u otras entidades, no asignando categoría genérica o específica alguna. Por lo tanto, se consideró no innovar en la denominación del material inventariado o referido, hasta que se resuelva la cuestión, lo que no implica un acuerdo a tal denominación. El material de $P$. venustula depositado en la colección del MACN corresponde a localidades de Chile.

Clase Bivalvia

Subclase Pteriomorphia Beurlen, 1944

Orden Mytiloidea Férussac, 1822

Fam. Mytilidae Rafinesque, 1815

Gen. Limnoperna Rochebrune, 1882

L. fortunei (Dunker, 1857); MLP= 5018, Ensenada (BA).

Gen. Mytella Soot-Ryen, 1955

M. charruana (d'Orbigny, 1842); MLP=2395, Punta Piedras (BA) y 2675.

Gen. Mytilus Linnaeus, 1758

M. edulis Linnaeus, 1758; MLP=1700, Punta Piedras (BA).

Comentarios: El material de colección bajo la denominación de Mytella falcata (d'Orbigny, 1846), se consideró sinónimo de M. charruana.

Subclase Paleoheterodonta Newell, 1965

Orden Unionoidea Stoliczka, 1871

Fam. Hyriidae Swaison, 1840

Gen. Castalia Lamarck, 1819

C. ambigua (d'Orbigny, 1835); MLP=1357, Gualeguaychú (ER).

C. psammoica (d'Orbigny, 1835); MLP=1413, Monte Hermoso (BA).

Gen. Diplodon Spix, 1827

D. (Diplodon) chilensis (Gray, 1828); MLP=1793, General Conesa (RN).

D. (D.) delodontus (Lamarck, 1819); MLP=3709, Romang (SF).

D. (D.) granosus (Bruguiere, 1792); sin material de referencia en colecciones.

D. (D.) parallelopipedon (Lea, 1834); MLP=1747, Iguazú (MS).

D. (D.) parodizi Bonetto, 1960; MLP=3710, Arroyo Seco (SF).

D. (D.) rhuacoicus (d'Orbigny, 1835); MLP= 6261, Concordia (ER); 6295; 6328 y 6387.

D. (D.) rotundus (Wagner, 1827); MLP=6664, Posadas (MS).

D. (D.) trifidus (Lea, 1860); MLP= 6351, Romang, (SF).

D. (Rhipidiodonta) charruanus (d'Orbigny, 1835); MLP=1760, Colón (ER).

D. (R.) hylaeus (d'Orbigny, 1835); MLP=3713, Puerto Malabrigo (SF).

D. (R.) variabilis (Maton, 1809); MLP=1754, Ensenada (BA).

Comentarios: Se siguió a Bonetto (1961) y a Bonetto y Tassara (1988) para el ordenamiento de las especies, ya que, además de las características valvares consideran las de los estadíos larvarios. Para la Argentina, C. ambigua, presentaría de sus dos subespecies sólo una, C. ambigua inflata (d'Orb., 1835). Diplodon (D.) granosus, estaría también representada por una sola subespecie $D$. (D.) granosus multistriatus (Lea, 1831). Diplodon (D.) rotundus, de sus subespecies se presenta $D$. (D.) rotundus rotundus. A su vez, $D$. (D.) delodontus presentaría tres subespecies: delodontus, expansus y waymanii. Por otro lado, una extensa lista de sinónimos específicos estarían comprendidos en $D$. (D.) chilensis y en $D$. (R.) charruanus. Diplodon (D.) parodizi, se distingue de $D$. (R.) variabilis y de $D$. (R.) charruanus (con desarrollo directo) ya que presenta un gloquidio parásito de peces. El material de colección observado y nominado como D. (D.) piceus (Lea, 1860) pasa a sinonimia de D. (D.) rhuacoicus. 
Fam. Etheriidae Swaison, 1840

Gen. Anodontites Bruguiére, 1792

A. (Anodontites) obtusus (d'Orbigny, 1835); $\mathrm{MLP}=5773$, Arroyo Tiguá (ER).

A. (A.) patagonicus (Lamarck, 1819); MLP=1262, Magdalena (BA).

A. (A.) puelchanus (d'Orbigny, 1835); MLP=1801, General Conesa (RN).

“A. (A.) soleniformis” (d’Orbigny, 1835); MLP=2669, Iguazú (MS).

A. (A.) tenebricosus (Lea, 1834); MLP=1786, San Javier (MS).

A. (A.) trapesialis (Lamarck, 1819); MLP=1258, Ensenada (BA).

A. (A.) trapezeus (d'Orbigny, 1835); MLP= 2013, Santo Tomé (SF).

A. (A.) trigonus (Gray, 1834); MLP= 5783, Río Nogoyá (ER).

A. (Lamproscapha) ensiformis (Spix \& Wagner, 1827); MLP=6663, Santa Fé (SF). MACN= 388.

Gen. Fossula Lea, 1870

F. fossiculifera (d'Orbigny, 1835); MLP=1775, Ituzaingó (CR); 5750 y 5844.

Gen. Leila Gray, 1840

L. blainvilleana (Lea, 1834); MLP=5756, Laguna Guadalupe (SF).

Gen. Monocondylea d'Orbigny, 1835

M. corrientesensis d'Orbigny, 1835; MLP= 3926, Paso de los Libres (CR).

M. minuana d'Orbigny, 1835; MLP=1780, Gualeguaychú (ER).

M. paraguayana d'Orbigny, $1835 ; \mathrm{MLP}=3706$, Río Colastiné (SF).

M. parchappii d'Orbigny, 1835; MLP= 5715, Corrientes (CR).

Gen. Mycetopoda d'Orbigny, 1835

M. legumen (Martens, 1828); MLP=2803, La Plata (BA).

M. siliquosa (Spix, 1827); MLP=209, Magdalena (BA).

M. soleniformis d'Orbigny, 1835; MACN= 545, Puerto Lujan (CR).

Comentarios: La nomenclatura de esta familia, tradicionalmente denominada Mycetopodidae, fue revisada por Kabat (1997). Para las entidades presentes en la Argentina de esta familia se siguió la revisión realizada por Castellanos y Landoni (1990). Anodontites (A.) trigonus sólo presentaría la subespecie A. (A.) trigonus georginae. De las cuatro subespecies de $A$ (A.) trapesialis, solamente se cuenta con la descrita como $A$. (A.) trapesialis susannae. Anodontites (A.) obtusus, cuenta con dos subespecies, de las cuales se registró solamente A. (A.) obtusus lucidus. Anodontites (A.) tenebricosus corresponde a la descrita por Bonetto (1967) como A. (A.) crispatus tenebricosus. Si bien Castellanos y Landoni (1990) sostienen a "A. (A.) soleniformis" como una especie válida, Bonetto y Ezcurra (1965) no consideran que sea ni especie ni subespecie válida, ya que encontraron formas graduales entre A. (A.) crispatus tenebricosus y A. (A.) crispatus soleniformis. Respecto a Ihringella isocardoides (Lea, 1857), los autores comentan que si bien el material tipo proviene del Río de la Plata, no ha vuelto a ser registrada (a pesar de las muy numerosas recolecciones realizadas en el río), y que posiblemente haya sido un error de procedencia.

Subclase Heterodonta Neumayr, 1884

Orden Veneroidea H. \& A. Adams, 1856

Fam. Corbiculidae Gray, 1847

Gen. Corbicula Mergele, 1811

C. fluminea (Müller, 1774); MLP= 4698, Atalaya (BA).

C. largillierti (Philippi, 1844); MLP= 4697, Punta Blanca (BA).

Gen. Neocorbicula Fischer, 1887

N. limosa (Maton, 1809); MLP=1776, Isla Martín García (BA).

N. paranensis (d'Orbigny, 1835); MLP=2397, Laguna Guadalupe (SF). 
Fam. Solecurtidae d'Orbigny, 1846

Gen. Tagelus Gray, 1847

T. gibbus (Splenger, 1794); MLP=1410, Monte Hermoso (BA).

Fam. Sphaeriidae Deshayes, 1855 (= Pisidiidae Gray, 1857)

Gen. Byssanodonta d'Orbigny, 1846

B. paranensis d'Orbigny, 1846; MLP=4694, Paso de la Patria (CR).

Gen. Eupera Bourguignat, 1854

E. platensis Doello Jurado, 1921; MLP= 5008, Empedrado (CR).

E. doellojuradoi Klappenbach, 1962; MLP= 5001, Monte Caseros (CR) y 8796.

E. guaraniana Ituarte, 1994; MLP=4998, Colón (ER) (Holotipo).

E. iguazuensis Ituarte, 1989; MLP= 4701, Iguazú (MS) (Tipo) y 4702 (Paratipos).

E. elliptica Ituarte \& Dreher-Mansur, 1993; MLP=4990, Iguazú (MS) (Holotipo).

E. klappenbachi Mansur \& Veitenheimer, 1975; MLP= 5313, Ayo Brazo Chico (ER).

Gen. Musculium Link, 1807

M. argentinum (d'Orbigny, 1835); MLP= 5016, Lago Fagnano (TF).

M. patagonicum Pilsbry, 1911; MLP=4989, Isla Gable (TF).

Gen. Pisidium Pfeiffer, 1821

P. chicha Ituarte, 2005; MLP= Entre Río Zapla y la ciudad Zapla 6899-1-1 (Holotipo).

P. chilensis (Gray, 1828); sin material de referencia en colecciones.

P. chiquitanum Ituarte, 2001; MLP= 5497, Terma de Reyes (JU).

P. dorbignyi (Clessin, 1879); MLP= 5512, Iguazú (MS); 5434.

P. inacayali Ituarte, 1996; MLP= 5066, Esquel (CH) (Holotipo).

P. magallanicum (Dall, 1908); MLP= 5054, Arroyo Chico (SC).

P. observationis Pilsbry, 1911; MLP= 4988, Isla Gable (TF); 5065; 5081; 5426 y 6964.

P. ocloya Ituarte, 2005; MLP= 5499-1, Río Burrumayo (JU) (Holotipo), 5499-2 (Paratipos)

P. omaguaca Ituarte, 2005; MLP= 5496-1-1, Río Reyes, Las Termas (JU) (Holotipo).

P. patagonicum Pilsbry, 1911; MLP= 5288, Lago Yehuín (TF).

P. pipoense Ituarte, 2000; MLP= 5336, La Cruz (CR) (Holotipo); 5298 (Paratipos).

P. plenilunium (Melville \& Standen, 1907); sin material de referencia en colecciones.

P. sterkianum Pilsbry, 1897; MLP= 5058, Ensenada (BA).

P. taraguyense Ituarte, 2000; MLP $=5318$ y 5347 La Cruz (CR) (Paratipos).

P. vile Pilsbry, 1897; MLP= 5062, Ensenada (BA); 5063; 5289; 5290 y 5294.

Gen. Sphaerium Scopoli, 1777

S. lauricochae (Philippi, 1869); MLP= 5087, San Salvador de Jujuy (JU) y 5088.

Orden Myoidea Golfuss, 1820

Fam. Erodonidae Winkworth, 1932

Gen. Erodona Bosc, 1801

E. mactroides Daudin, 1802; MLP=1889, Ensenada (BA). 


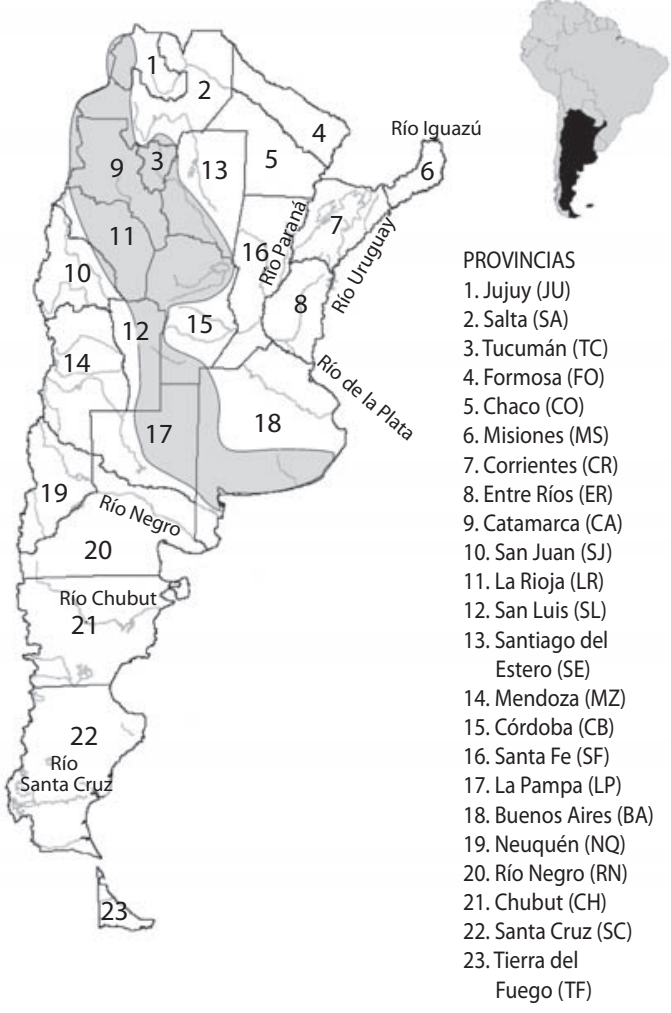

Fig. 1. República Argentina con los principales ríos y provincias. Área gris: Zona de Transición.

Fig. 1. Main rivers and provinces from Argentina Republic. Gray area: Transition zone.

\section{DISCUSIÓN}

Consideraciones generales: hasta el presente han sido descritas 166 especies. De ellas 101 pertenecen a 10 familias de Gastropoda y 65 a 7 de Bivalvia. Las familias de mayor riqueza en especies son los Lithoglyphidae (22) y Sphaeriidae (25) respectivamente. El Cuadro 2 muestra las especies presentes en cada una de las provincias que conforman la Argentina.

- Ampullariidae (Fig. 2A). Las especies de esta familia se distribuyen en el centro y norte del país. Posee una sola especie endémica de la Argentina Pomella (P.) americanista, que se ha registrado en los márgenes de los ríos Alto Paraná y
Uruguay. La especie de más amplia distribución es Pomacea (P.) canaliculata, muy frecuente en los cuerpos de agua de toda el área central y norte del país.

- Thiaridae (Fig. 2B). Hasta el presente existen tres especies endémicas, $A$. guaraniticum, A. chloroticum y A. stigmaticum, que pueden ser consideradas extintas en sus hábitats naturales (Quintana y Mercado Laczkó 1997). Sin embargo, ellas no figuran como especies CITES en la Lista Roja de la IUCN. Estas especies son endémicas del río Alto Paraná, y de las áreas de influencia de la represa Yacyretá, provincia de Corrientes, cuya construcción ha modificado profundamente su hábitat natural, transformando un río correntoso y con numerosos saltos en un embalse. La especie restante, M. tuberculatus, es de introducción reciente en el nordeste argentino (ver ítem "Especies Exóticas").

- Cochliopidae (Fig. 2C). De amplia distribución en la Argentina. Diez de sus especies presentes son endémicas: $H$. castellanosae, H. conexa, H. kuesteri, $H$. montana, $H$. occidentalis, $H$. peiranoi, $H$. rionegrina, $H$. sublineata, $H$. tucumana, $H$. vianai. Todas ellas presentan solamente uno o dos registros. $H$. parchappii es la de mayor distribución en el norte y centro del país, mientras que $H$. hatcheri se distribuye principalmente en la región patagónica.

- Lithoglyphidae (Fig. 2D). Excepto por Potamolithus valchetensis, endémica de la meseta de Somuncurá, provincia de Río Negro (Patagonia), las especies de esta familia se distribuyen por las cuencas de los ríos Uruguay y Alto Paraná. Algunas de ellas presentan registros puntuales: $P$. valchetensis, $P$. tricostatus, $P$. rushii, $P$. quadratus, P. hidalgoi, P. dinochilus, $P$. concordianus. Estas especies, a excepción de $P$. quadratus, más $P$. agapetus, $P$. conicus, $P$. paranensis, $P$. philippianus y $P$. simplex son endémicas.

- Glacidorbidae (Fig. 2B). Según Ponder y Avern (2000) esta familia presentan un patrón de distribución gondwánico 


\section{CUADRO 1}

Material examinado en las distintas colecciones: MLP (Museo de La Plata); MACN (Museo Argentino de Ciencias Naturales); FML (Fundación Miguel Lillo)

\section{TABLE 1}

Material examined in the different collections: (Museo de La Plata); MACN (Museo Argentino de Ciencias Naturales); FML (Fundación Miguel Lillo)

Especie

Ampullariidae

A. platae

A. puelchella

A. spixii

F. neritiniformis

F. iheringi

M. planogyra

P. (P.) canaliculata

P. (P.) insularum

P. (P.) scalaris

P. (P.) americanista

P. (P.) megastoma
Números de Colección

$\mathrm{MLP}=364 ; 7638$ al 7647; 26190; 26192; 26194.

$\mathrm{MLP}=1948 ; 2121 ; 4178 ; 7648$ al 7661; 26177.

$\mathrm{MACN}=489 ; 559 ; 5191 ; 5263 ; 6401 ; 6431 ; 9391 ; 9520 ; 9525 ; 9552 ; 9557 ; 11535 ; 11938 ; 12890$; $12974 ; 13966 ; 14051 ; 14640 ; 14729 ; 14875 ; 18230 ; 19180 ; 20075 ; 21049 ; 25581 ; 25587 ; 25817$; 25934; $26740 ; 26772 ; \mathrm{S} / \mathrm{N}^{\circ}=4$.

$\mathrm{FML}=612$

$\mathrm{MLP}=362 ; 5272 ; 6448 ; 6449 ; 6713 ; 7079 ; 7669$ al 7680; 26088.

$\mathrm{FML}=312 ; 737$

$\mathrm{MACN}=1005 ; 11902 ; 17100$.

$\mathrm{MACN}=6255 ; 14527 ; 16388 ; 17100 ; 17343 ; 17353 ; 26710 ; 32717 ; 145276$.

$\mathrm{MLP}=2122 ; 2123 ; 3630 ; 7230 ; 9394 ; 9396$ al 9398; 26246; 26247.

$\mathrm{MACN}=1350 ; 1917 ; 2824 ; 5946 ; 9486 ; 12183 ; 12973 ; 14641 ; 14877 ; 19145 ; 20020 ; 29592$.

$\mathrm{MLP}=283 ; 285 ; 286 ; 316$ al 318; 320; 322; 332 al 334; 337; 339; 340; 342 al 355; 367; 740; 1104; $1216 ; 1225 ; 1304 ; 1368 ; 2116 ; 2170 ; 2274 ; 2306 ; 2519 ; 2807 ; 3348$ al $3353 ; 3358 ; 3361$ al 3373 ; $3445 ; 3447 ; 3685 ; 3687 ; 3817 ; 4183 ; 4446 ; 4451 ; 5278 ; 5639 ; 6265 ; 6337 ; 6338 ; 6340$ al 6342 ; 6438; 6440 al 6442; 6686; 6741; 6997; 7019; 7020; 7032; 7077 al 7080; 7082; 7084 al 7086; 7088; 7090; 7092 al 7105; 7216; 7217; 7347; 9858 al 9905; 10933; 10937; 11359; 11584; 11588; 11589; $11605 ; 11609 ; 11716 ; 11717 ; 11750 ; 11774 ; 11807 ; 11881 ; 11886 ; 26004 ; 26028 ; 26029 ; 26041$; $26065 ; 26076 ; 26086$ al 26093; 26226.

MACN $=401 ; 557 ; 722 ; 1048 ; 1957 ; 2465 ; 4306 ; 4422 ; 5254 ; 5354 ; 6370 ; 6412 ; 8805 ; 9200$; 9391; 9486; 9570; 9657; 9727; 10021; 10424; 11809; 12087; 12191; 14073; 14527; 14575; 14731; $15464 ; 15710 ; 15713 ; 15955 ; 17477 ; 18315 ; 18360 ; 19396 ; 19519 ; 19521 ; 20879 ; 24723 ; 25786$; 26535 al 26537; 26540; 26705; 26707; 27241; 28582; 28584; 28589; 28597; 28599; 32950; $\mathrm{S} / \mathrm{N}^{\circ}=$ 6.

$\mathrm{FML}=33 ; 50 ; 101 ; 102 ; 165 ; 210 ; 223 ; 224 ; 329 ; 476 ; 514 ; 515 ; 546 ; 610 ; 611 ; 808 ; 817 ; 847$; $871 ; 872 ; 877 ; 880 ; 1620 ; 5780$ al 5782;6515; 10941; 11057.

$\mathrm{MACN}=9391 ; 9570 ; 9701 ; 9734 ; 13997 ; 14733 ; \mathrm{S} / \mathrm{N}^{\mathrm{o}}=1$.

$\mathrm{FML}=5784$

$\mathrm{MLP}=3356 ; 5638 ; 6443 ; 6855 ; 7079 ; 9912$ al 9926; 10940; 11585; 11595; 11723; 11882; 11885; $26092 ; 26221 ; 26225 ; 26227$.

$\mathrm{MACN}=6815 ; 9734 ; 11534 ; 11838 ; 12970 ; 14068 ; 14642 ; 14727 ; 14728 ; 14878 ; 14924 ; 16328$; $19160 ; 19181 ; 20876 ; 20950 ; 21050 ; 24722 ; 29186 ; \mathrm{S} / \mathrm{N}^{\circ}=4$.

$\mathrm{FML}=53 ; 106 ; 625 ; 630$.

$\mathrm{MLP}=368 ; 5142$ al $5144 ; 5153 ; 7279 ; 7280 ; 7624$ al 7631 .

$\mathrm{MACN}=17191 ; 21788 ; 25824 ; \mathrm{S} / \mathrm{N}^{\circ}=1$

$\mathrm{FML}=107 ; 228 ; 5783$

$\mathrm{MLP}=237$ al $329 ; 331 ; 379 ; 1271 ; 5257 ; 5510 ; 5636 ; 5641 ; 5668 ; 5687 ; 5688 ; 5707 ; 6445 ; 6446$; $6700 ; 6702 ; 6703 ; 6996 ; 7002 ; 7082 ; 7091 ; 7633 ; 7634 ; 7636 ; 7637 ; 10941 ; 11600 ; 26143 ; 26214$; $26216 ; 26217$.

MACN=9047; 9260; 9515; 17354; 20515.

$\mathrm{FML}=108$ 
CUADRO 1 (Continuación...)

Material examinado en las distintas colecciones: MLP (Museo de La Plata); MACN

(Museo Argentino de Ciencias Naturales); FML (Fundación Miguel Lillo)

TABLE 1 (Continued...)

Material examined in the different collections: (Museo de La Plata); MACN

(Museo Argentino de Ciencias Naturales); FML (Fundación Miguel Lillo)

Especie

Cochliopidae

H. australis

H. castellanosae

H. conexa

H. hatcheri

H. kuesteri

H. parchappii

H. piscium

H. tucumana

Lithoglyphidae

P. agapetus

P. buschii

P. doeringi

P. iheringi

P. lapidum

P. orbignyi

P. paranensis

P. peristomatus

P. petitianus
Números de Colección

MLP= 1392; 4327; 6918; 9137 al 9141; 11744 .

$\mathrm{MACN}=9712 ; 10678 ; 11334 ; 13849 ; 20284 ; 22472 ; 28621 ; 28793 ; 28881 ; 28883 ; 29796$.

$\mathrm{MLP}=4022 ; 4025$.

$\mathrm{MLP}=3685 ; 4031 ; 4043 ; 4047 ; 4241$.

$\mathrm{MLP}=2298 ; 4002 ; 4423 ; 5509 ; 9101 ; 9146$ al $9148 ; 9167$ al 9175.

$\mathrm{MACN}=950 ; 9538 ; 21770$.

$\mathrm{MACN}=19105 ; 20997 ; 28633 ; 30244$.

$\mathrm{MLP}=381 ; 664 ; 2113 ; 2297 ; 2304 ; 6697 ; 6698 ; 6708 ; 7106 ; 7107 ; 9154$ al 9159.

$\mathrm{MACN}=9481 ; 10121 ; 11562 ; 12122 ; 14576 ; 16402 ; 16524 ; 18630 ; 21120 ; 28613 ; 28622 ; 28795$; $29360 ; 29392 ; 30592 ; 30632$.

MLP= 6780; 6794; 7108; 7109; 9091; 9162 al 9164.

$\mathrm{MACN}=9489 ; 10391 ; 10911 ; 11841 ; 11885 ; 13995 ; 14234 ; 16420 ; 28610 ; 28641 ; 28794 ; 29328$; $29331 ; 30241$.

$\mathrm{FML}=1298$.

$\mathrm{MLP}=4012 ; 4036$.

$\mathrm{FML}=1431$ (Paratipos).

$\mathrm{MLP}=4628 ; 4652 ; 4986 ; 6760 ; 6763$ al 6766; 6768; 6770; 6771; 6773; 6775; 6776; 6779; 6780; $6785 ; 6790 ; 6793 ; 6796$.

$\mathrm{MACN}=2729 ; 9515 ; 10438 ; 14735 ; 25935$.

$\mathrm{MLP}=4623 ; 4644 ; 4645 ; 4650 ; 6761 ; 6762 ; 6767 ; 6769 ; 6772 ; 6774 ; 6777 ; 6778 ; 6782$ al $6784 ;$ $6786 ; 6787 ; 6789 ; 6791 ; 6792 ; 6794 ; 6795 ; 6797$.

$\mathrm{MACN}=604 ; 9511 ; 9515 ; 10438 ; 12945 ; 14735 ; 17111 ; 17335 ; 17336 ; 17344 ; 25935 ; 27291 ; \mathrm{S} /$ $\mathrm{N}^{\mathrm{o}}=1$.

$\mathrm{MACN}=360 ;$ Lote $\mathrm{S} / \mathrm{N}^{\mathrm{o}}=1$.

$\mathrm{MACN}=17335 ; 25792 ; 25794$.

$\mathrm{MLP}=4615 ; 4621 ; 4623 ; 4629 ; 4645 ; 4650 ; 4652 ; 6788 ; 6996$ al $6999 ; 7001 ; 7002 ; 7004 ; 9944$ al 9947.

$\mathrm{MACN}=604 ; 9341 ; 9515 ; 9541 ; 10438 ; 10910 ; 11905 ; 12945 ; 14735 ; 16419 ; 17111 ; 17334$ al $17336 ; 17338$ al $17340 ; 17349$ al $17351 ; 25809 ; 25855 ; 25935 ; 27291 ; \mathrm{S} / \mathrm{N}^{\mathrm{o}}=2$. .

$\mathrm{MACN}=9515 ; 14735 ; 25792 ; 25794$.

$\mathrm{MLP}=4620 ; 4624 ; 4627 ; 4629 ; 4634$.

$\mathrm{MACN}=25809 ; 25855 ; 19154$.

$\mathrm{MLP}=4619 ; 4620 ; 4624 ; 4626 ; 4646 ; 4649$.

$\mathrm{MACN}=19154$

$\mathrm{MACN}=8844 ; 9512 ; 9515 ; 10438 ; 14735 ; 17111 ; 17340 ; 17336 ; 17337 ; 19945 ; 25935 ; 27291$. 
CUADRO 1 (Continuación...)

Material examinado en las distintas colecciones: MLP (Museo de La Plata); MACN

(Museo Argentino de Ciencias Naturales); FML (Fundación Miguel Lillo)

TABLE 1 (Continued...)

Material examined in the different collections: (Museo de La Plata); MACN

(Museo Argentino de Ciencias Naturales); FML (Fundación Miguel Lillo)

Especie
P. philipianus
P. valchetensis
Chilinidae
C. dombeiana
C. fluminea
C. fulgurata
C. gallardoi
C. gibbosa

C. patagonica

C. rushii

Lymnaeidae

"L." columella

L. diaphana

L. viatrix

Planorbidae

A. nordestensis
Números de Colección

$\mathrm{MLP}=4622 ; 4629 ; 4630 ; 4632 ; 7004 ; 7007,7008$.

$\mathrm{MACN}=17110 ; 17344 ; 25789$ al 25790.

$\mathrm{MACN}=3412$ y 3413 (Paratipos).

$\mathrm{MLP}=770 ; 1916 ; 2902 ; 2907 ; 2915 ; 5253 ; 8240$.

$\mathrm{MACN}=25895 ; 28823 ; 30605$.

$\mathrm{MLP}=773 ; 2488 ; 2629 ; 5246 ; 5261 ; 8235 ; 8236 ; 8241$ al $8245 ; 8247 ; 11773$.

$\mathrm{MACN}=590 / 91 ; 9272 ; 9375 ; 9512 ; 9530 ; 9542 ; 9583 ; 9927 ; 9933 ; 9954 ; 10370 ; 11738 ; 12121$; $12944 ; 14136 ; 14734 ; 14754 ; 15463 ; 15477 ; 19814 ; 19972 ; 20185 ; 24303 ; 28670 ; 28675 ; \mathrm{S} / \mathrm{N}^{\mathrm{o}}=5$.

$\mathrm{MLP}=4103 ; 4104 ; 5508 ; 8250$.

$\mathrm{MACN}=1990 ; 9040 ; 14450 ; 20172 ; 20993 ; 25873 ; \mathrm{S} / \mathrm{N}^{\mathrm{o}}=1$.

MLP= 11067 (Paratipo); 4451; 8237; 8252 .

$\mathrm{MACN}=8241 ; 17103 ; 17104 ; 17196 ; 17345 ; 25801 ; 32798$.

$\mathrm{MLP}=728 ; 2127 ; 2917 ; 2918 ; 2920 ; 3827 ; 4097 ; 4125 ; 8253$ al $8258 ; 29415$.

$\mathrm{MACN}=6770 ; 6771 ; 9063 ; 9346 ; 9680,9775 ; 9961 ; 10272 ; 13371,13758 ; 19058 ; 19096 ; 19118$; $20178 ; 20278 ; 21763 ; 21795 ; 21796 ; 23298 ; 25881 ; 26504 ; 30634 ; 30815 ; 32641 ; \mathrm{S} / \mathrm{N}^{\mathrm{o}}=1$.

$\mathrm{MLP}=11069$ (Paratipo); 5098; 5099; 5128 al 5130; 5210; 7316.

MLP $=765 ; 766 ; 768 ; 1221 ; 2125 ; 2132 ; 2287 ; 2904 ; 2909 ; 4098 ; 6988 ; 6989 ; 7358$ al $7360 ; 8263$ al $8273 ; 29445 ; 29451$.

MACN $=995 ; 1643 ; 4221 ; 8451 ; 9120 ; 9268 ; 9368 ; 9874 ; 9925 ; 9926 ; 9928 ; 9931 ; 9932 ; 11089$; $11722 ; 11884 ; 12721 ; 13033 ; 13099 ; 13372 ; 14550 ; 15449 ; 16525 ; 17084 ; 17417 ; 17692 ; 18246$; $20990 ; 21182 ; 21265 ; 21782 ; 26283 ; 29363 ; 29385 ; 32643 ; 34062$.

$\mathrm{MLP}=278 ; 2905 ; 2908 ; 12587$.

$\mathrm{MACN}=12586 ; 33655$.

$\mathrm{MACN}=9341 ; 28674 ; 28678$.

$\mathrm{MLP}=9235 ; 5657 ; 7275$ al 7277; 7366; 10023 .

$\mathrm{MACN}=28068 ; 29324$.

$\mathrm{MLP}=9236$ al 9238; 9240; 9241; 9243; 12582.

$\mathrm{MACN}=10140 ; 12580 ; 12583 ; 14231 ; 29356$.

$\mathrm{FML}=1992 ; 3238 ; 3239 ; 10540$.

$\mathrm{MLP}=787 ; 2111 ; 2112 ; 2266 ; 3541 ; 4469 ; 5021 ; 9352$ al 9362; 11743.

$\mathrm{MACN}=2402 ; 9779 ; 9938 ; 10131 ; 10901 ; 11090 ; 11115 ; 11561 ; 11836 ; 12893 ; 14505 ; 14518$, 14864; 15446; 15645; 20282; 20991 al 20996; 23085; 25438; 26591; 28665 al 28667; 30492; 30768.

$\mathrm{FML}=508 ; 518 ; 521 ; 540 ; 590 ; 991 ; 1031 ; 2392 ; 2393 ; 10687 ; 11003$.

$\mathrm{MLP}=7278 ; 7602 ; 8545$ al 8549 
CUADRO 1 (Continuación...)

Material examinado en las distintas colecciones: MLP (Museo de La Plata); MACN

(Museo Argentino de Ciencias Naturales); FML (Fundación Miguel Lillo)

TABLE 1 (Continued...)

Material examined in the different collections: (Museo de La Plata); MACN

(Museo Argentino de Ciencias Naturales); FML (Fundación Miguel Lillo)

Especie

A. petricola

B. peregrina

B. straminea

B. tenagophila

D. anatinum

D. cimex

D. depressissimun

D. kermatoides

D. lucidum

Ancylidae

A. obliquus

H. moricandi

U. concentricus

Physidae

"P. cubensis"
Números de Colección

$\mathrm{MLP}=5090 ; 5122$ al 5125; 5517; 5522.

$\mathrm{MLP}=782 ; 1496 ; 2141 ; 2259 ; 2883 ; 5127 ; 5319 ; 6807 ; 7009 ; 7011$ al $7013 ; 7015 ; 7017 ; 7018$; $7021 ; 7025 ; 7031 ; 7044 ; 7058 ; 7177 ; 7357 ; 7432 ; 7789$ al $7798 ; 9839 ; 10720$ al $10724 ; 10785$ al $10799 ; 11860$.

MACN $=226 ; 283 ; 373 ; 487 ; 801 ; 834 ; 936 ; 937 ; 939 ; 952 ; 1399 ; 1625 ; 1759 ; 1972 ; 1999 ; 2119$; 2399; 2630; 6921; 8591; 9120; 9308; 9446; 9520; 9525; 9554; 9574; 9593; 9778; 9863; 9945; $9953 ; 10117 ; 10275 ; 10386 ; 10425 ; 10507 ; 10654 ; 11088 ; 11566 ; 11698 ; 11715 ; 11716 ; 11753$; $11759 ; 12896 ; 13079 ; 13102 ; 14206 ; 14235 ; 14600 ; 14664 ; 14666 ; 14723 ; 14901 ; 15471 ; 15612$; $16089 ; 16097 ; 16403 ; 16415 ; 17324 ; 17420 ; 17690 ; 18357 ; 18629 ; 19111 ; 19584 ; 20048 ; 20102$; 20280; 20880; 21913; 23088; 23212; 23852; 24310; 26218; 26544; 26686; 27209; 29372; 95014. $\mathrm{FML}=331 ; 516 ; 613 ; 682 ; 745 ; 937 ; 997 ; 2651 ; 2653 ; 3135 ; 3139 ; 11022$.

$\mathrm{MLP}=7015 ; 7026 ; 7039 ; 7043 ; 7046 ; 7057 ; 7059 ; 7180 ; 7181 ; 7852 ; 7855 ; 7856 ; 10781 ; 11783$ $11818 ; 30031$.

$\mathrm{MACN}=598 ; 1197 ; 9246 ; 9520 ; 13102 ; 15471 ; \mathrm{S} / \mathrm{N}^{\mathrm{o}}=3$.

$\mathrm{MLP}=775 ; 776 ; 778 ; 781 ; 2145 ; 2256 ; 2886 ; 4442 ; 4611 ; 5320 ; 5326 ; 5640 ; 6800 ; 7005 ; 7010$; $7015 ; 7016 ; 7022$ al 7024; 7026 al 7029; 7038; 7045 al 7047; 7148; 7163; 7164; 7178; 7179; 7182; 7699 al $7703 ; 7857$ al $7869 ; 11742 ; 11755 ; 11763 ; 11765 ; 11766 ; 11768 ; 11780 ; 11806 ; 11811$ al $11813 ; 11840 ; 11884 ; 11887$.

$\mathrm{MACN}=226 ; 470 ; 513 ; 719 ; 9375 ; 11055 ; 13079 ; 14600 ; 14666 ; 16096 ; 17325 ; 18603 ; 19144$; $20075 ; 20077 ; 26501 ; 26597 ; 29374 \mathrm{~S} / \mathrm{N}^{\mathrm{o}}=3$.

$\mathrm{FML}=131 ; 148 ; 391 ; 886 ; 2654 ; 3137 ; 10602 ; 11905$.

$\mathrm{MLP}=7026 ; 7030 ; 7033 ; 7165 ; 7166 ; 7168$ al 7170; 7346; 7348; 8527; 11808 . $\mathrm{MACN}=28544$.

$\mathrm{MLP}=6998 ; 7015 ; 7039 ; 7143 ; 7174$ al 7176; 11809; 11814; 11816.

$\mathrm{MLP}=7389 ; 8531 ; 8532$.

$\mathrm{MACN}=9391 ; 26534$.

$\mathrm{MLP}=2260 ; 6798 ; 7009 ; 7014 ; 7015 ; 7024 ; 7030 ; 7034 ; 7039 ; 7040 ; 7043 ; 7171$ al 7173; 7218; 8539 al $8542 ; 11778$.

$\mathrm{MACN}=598 ; 784 ; 939 ; 1911 ; 2400 ; 9574 ; 9710 ; 9711 ; 16414 ; 19516 ; 20077 ; 21289 ; 27326$; 29358; 32794. $\mathrm{S} / \mathrm{N}^{\circ}=3$.

$\mathrm{MLP}=7041 ; 7042 ; 7144 ; 7149$ al 7155; 7157 al 7161; 7388; 8544; 11756; 11820.

$\mathrm{MACN}=9037$.

$\mathrm{MLP}=4534 ; 4538 ; 4544 ; 7037$.

$\mathrm{MLP}=3611 ; 4532 ; 4537 ; 4539 ; 4542 ; 4550 ; 4552 ; 4553 ; 4556 ; 4557 ; 7156 ; 7160 ; 7327$ al 7339 ; 8937 al $8942 ; 11777 ; 11863 ; 11874 ; 30601$.

$\mathrm{MLP}=2160 ; 4531 ; 4533 ; 4535 ; 4536 ; 4540 ; 4541 ; 4543 ; 4547 ; 4548 ; 4555 ; 4559 ; 4563 ; 4564$; $4567 ; 5327 ; 7035 ; 7036 ; 7340$ al $7345 ; 30603$.

$\mathrm{MLP}=6521 ; 6522 ; 6925$ al 6930; 11839; 11841; 11843; 11846; 11848 
CUADRO 1 (Continuación...)

Material examinado en las distintas colecciones: MLP (Museo de La Plata); MACN

(Museo Argentino de Ciencias Naturales); FML (Fundación Miguel Lillo)

TABLE 1 (Continued...)

Material examined in the different collections: (Museo de La Plata); MACN

(Museo Argentino de Ciencias Naturales); FML (Fundación Miguel Lillo)

Especie

"S. marmorata"

\section{BIVALVIA}

Mytilidae

L. fortunei

M. charruana

M. edulis

Hyriidae

C. ambigua

C. psammoica

D. chilensis

D. delodontus

D. parallelopipedon

D. parodizi

D. charruanus

D. hylaeus

D. variabilis
Números de Colección

$\mathrm{MLP}=4441 ; 4451 ; 7191 ; 7192 ; 7317$ al $7326 ; 10934 ; 11782 ; 11817 ; 11819$.

MACN $=491 ; 596 ; 2401 ; 9035 ; 9226 ; 9269 ; 9594 ; 9722 ; 9827 ; 9936 ; 9937 ; 9953 ; 9954 ; 10018$; $10600 ; 10931 ; 11114 ; 12132 ; 12240 ; 14724 ; 14862 ; 15206 ; 15482 ; 16400 ; 18176 ; 18632 ; 20031$; 20954; 22359; 22929; 23561; 25856; 26128; 26129; 26569; 28300; 28370; 28574 al 28576; 28605 ; $28732 ; 29359 ; 29366 ; 29370 ; 29375 ; 30184 ; 30185$ al 30202.

$\mathrm{FML}=250 ; 520 ; 550 ; 775 ; 957 ; 1971 ; 10936$.

$\mathrm{MLP}=5136 ; 5170 ; 5203 ; 5205$ al 5207; 5279; 5285 al 5287; 5340; 5364; 5365; 5424; 5518; 5644; $6264 ; 6662 ; 6903 ; 7118 ; 7184$.

$\mathrm{MACN}=9178 ; 29150$.

$\mathrm{MLP}=2844 ; 4917$ al 4919; 50057; 50058; 50061.

$\mathrm{FML}=695 ; 708 ; 2425 ; 2426 ; 10843$.

$\mathrm{MLP}=1772 ; 1779 ; 1783 ; 2668 ; 2688 ; 2773 ; 3705 ; 3708 ; 3699 ; 5265 ; 6076 ; 6077 ; 6122 ; 6125$; $6129 ; 6131 ; 6144 ; 6150 ; 6157 ; 6182 ; 6192$ al $6194 ; 6197 ; 6198 ; 6202 ; 6205 ; 6206 ; 6232 ; 6313$; $6376 ; 6377 ; 6414$ al $6416 ; 6467$ al $6469 ; 6471 ; 6473 ; 6853 ; 7020 ; 8208 ; 8210 ; 8211$. $\mathrm{MACN}=6319$.

MLP $=3711 ; 6127 ; 6183 ; 6204 ; 6216 ; 6221 ; 6409 ; 6463 ; 8212$.

$\mathrm{MACN}=602 ; 5124,5194 ; 6308 ; 9432 ; 10223 ; 12194 ; 14456 ; 14638 ; 14699 ; 16408 ; 16720$; $16835 ; 18223 ; 19191 ; 19288 ; 23308 ; \mathrm{S} / \mathrm{N}^{\circ}=1$.

$\mathrm{MLP}=1794 ; 1795 ; 1798$ al $1800 ; 1802 ; 1803 ; 1903 ; 1904 ; 2496 ; 2504 ; 2794 ; 2796 ; 2797 ; 2799$; $2800 ; 2804 ; 3821 ; 3822 ; 6367 ; 6425 ; 7113 ; 7119$.

$\mathrm{MLP}=2793 ; 3697 ; 6124 ; 6154 ; 6211 ; 6238 ; 6242 ; 6246 ; 6250 ; 6259 ; 6347 ; 6353 ; 6369 ; 6436$; $6668 ; 8469 ; 11791$.

$\mathrm{MACN}=11102$.

$\mathrm{MLP}=1748$ al $1751 ; 1755 ; 2014 ; 2015 ; 3691 ; 3700 ; 6034$ al 6036; 6039; 6040; 6062; 6066; 6132; $6147 ; 6151 ; 6254 ; 6257 ; 6378 ; 6566 ; 6591 ; 6596 ; 7050 ; 8473 ; 10950$.

$\mathrm{MACN}=1923 ; 3106 ; 6429 ; 7789 ; 8772 ; 9376 ; 9485 ; 9491 ; 9604 ; 10259 ; 11101 ; 11731 ; 13759$; 14647; 14678; 14707; 17307; 17311; 17469; 19409; 21471; 21616; 25518; 25816; 28466 al 28468 ; $\mathrm{S} / \mathrm{N}^{\mathrm{o}}=4$.

$\mathrm{MLP}=3714 ; 6112 ; 6224 ; 6225 ; 6236 ; 6240 ; 6243 ; 6244 ; 6247 ; 6255 ; 6258 ; 6260 ; 6375 ; 6454$; $6564 ; 6615$.

$\mathrm{MACN}=18218$

$\mathrm{MLP}=3698 ; 3702 ; 3703 ; 3715 ; 6075 ; 6126 ; 6141 ; 6156 ; 6167 ; 6327 ; 6427 ; 6563 ; 6604 ; 8466$.

$\mathrm{MLP}=6179 ; 6267 ; 6293 ; 6310 ; 6326 ; 6355 ; 6383 ; 6402 ; 6419 ; 6421$.

$\mathrm{MLP}=1762 ; 1767 ; 1768 ; 6045 ; 6050 ; 6133$ al $6137 ; 6142 ; 6210 ; 6226 ; 6234 ; 6235 ; 6241 ; 6249$; $6252 ; 6299 ; 6308 ; 6321 ; 6352 ; 6358 ; 6371 ; 6404 ; 6434 ; 6525 ; 6611 ; 6623 ; 6997 ; 11787$.

$\mathrm{MACN}=6332$. 
CUADRO 1 (Continuación...)

Material examinado en las distintas colecciones: MLP (Museo de La Plata); MACN

(Museo Argentino de Ciencias Naturales); FML (Fundación Miguel Lillo)

TABLE 1 (Continued...)

Material examined in the different collections: (Museo de La Plata); MACN

(Museo Argentino de Ciencias Naturales); FML (Fundación Miguel Lillo)

Especie

Etheriidae

A. obtusus

A. patagonicus

A. puelchanus

"A. soleniformis"

A. tenebricosus

A. trapesialis

A. trapezeus

A. trigonus

L. blainvilleana

M. corrientesensis

M. minuana

M. paraguayana

M. legumen

M. siliquosa
Números de Colección

$\mathrm{MLP}=5784 ; 5802 ; 5814 ; 5843 ; 5849 ; 5855 ; 5933 ; 6020 ; 6592$. $\mathrm{MACN}=11950$.

$\mathrm{MLP}=1771 ; 1775 ; 1787 ; 1790 ; 1910 ; 2658 ; 2660 ; 2664 ; 2792 ; 2793 ; 5270 ; 5761 ; 5774 ; 5787$; $5791 ; 5808 ; 5818 ; 5824 ; 5829 ; 5832 ; 5837 ; 5869 ; 5885 ; 5940 ; 5951 ; 5965 ; 5970 ; 5977 ; 5982$; $5985 ; 5994 ; 5996 ; 5998 ; 6022 ; 6024 ; 6029 ; 6030 ; 6484 ; 6579 ; 6583 ; 6852 ; 6997 ; 7020 ; 7571$ al 7578 .

$\mathrm{MACN}=10216 ; 30363 ; 30384$.

$\mathrm{FML}=8 ; 65 ; 288 ; 638 ; 11210$.

$\mathrm{MLP}=4154 ; 4155 ; 5360 ; 5361 ; 5973 ; 6016$.

$\mathrm{MACN}=5258 ; 10271$.

$\mathrm{MLP}=1775 ; 5388 ; 5769 ; 7579 ; 5794 ; 5857$.

$\mathrm{MACN}=498 ; 558 ; 6418 ; 14689 ; 14692 ; 14697 ; 19151 ; 19515$.

$\mathrm{MLP}=1789 ; 3966 ; 5790 ; 5816 ; 5848 ; 5868 ; 5937 ; 6025 ; 6580 ; 7020 ; 7581 ; 7582$.

$\mathrm{MACN}=10522$.

$\mathrm{FML}=49 ; 184$.

$\mathrm{MLP}=1250 ; 1261 ; 1267 ; 1325 ; 1773 ; 1784 ; 1785 ; 2013 ; 2277 ; 2655 ; 2658 ; 2660 ; 2661 ; 2663$; $2664 ; 2798 ; 5269 ; 5752 ; 5781 ; 5782 ; 5785 ; 5789 ; 5793 ; 5817 ; 5819 ; 5826 ; 5853 ; 5884 ; 5902$; $5930 ; 5936 ; 5949 ; 5963 ; 5969 ; 5978 ; 5993 ; 5995 ; 6014 ; 6018 ; 6031.6569 ; 6578 ; 6586 ; 6670$; $6941 ; 7215 ; 7219 ; 7583$ al $7594 ; 11888$.

$\mathrm{MLP}=5268 ; 5753 ; 5757 ; 5758 ; 5813 ; 5854 ; 5917 ; 5918 ; 5928 ; 5939 ; 5950 ; 5964 ; 6013 ; 6028$; $6581 ; 7595$.

$\mathrm{MACN}=5125$ al 5127; 9468; 9569; 9602; 10218; 11926; 11951; 12963; 12983; 17303; 19174; $19260 ; 21474$.

$\mathrm{MLP}=5800 ; 5840 ; 5916 ; 5962 ; 7020 ; 7597$.

$\mathrm{MLP}=5887 ; 5891 ; 5988 ; 5901 ; 6015 ; 9121 ; 9123$.

$\mathrm{MACN}=6287 ; 6342 ; 7782 ; 9603 ; 10265 ; 11536 ; 11943 ; 16987 ; 18227 ; 25983 ; \mathrm{S} / \mathrm{N}^{\mathrm{o}}=1$.

$\mathrm{FML}=667$.

$\mathrm{MLP}=4388 ; 5724 ; 5732 ; 5792 ; 5812 ; 5867 ; 5958 ; 9435 ; 9436$.

$\mathrm{MLP}=4082 ; 5730 ; 5735 ; 5786 ; 5822 ; 5834 ; 5957 ; 6019$.

$\mathrm{MLP}=3927 ; 4389 ; 5711 ; 5722 ; 5733 ; 5788 ; 5809 ; 5828 ; 5833 ; 5938 ; 5972 ; 5974 ; 6582$.

$\mathrm{MLP}=5807 ; 5830 ; 5976 ; 5981 ; 6021 ; 6483 ; 6585 ; 6593 ; 6669 ; 6684 ; 11792 ; 60011$.

$\mathrm{MACN}=473 ; 1731 ; 6427 ; 8417 ; 8723 ; 9468 ; 9491 ; 9606 ; 10221 ; 10261 ; 10902 ; 11881 ; 11935$; $11937 ; 16986 ; 17321 ; 19126 ; 20090 ; 21465 ; 21472 ; \mathrm{S} / \mathrm{N}^{\mathrm{o}}=1$.

$\mathrm{MLP}=1774 ; 2506 ; 5754 ; 5759 ; 5760 ; 5764 ; 5767 ; 5768 ; 5777 ; 5797 ; 5798 ; 5801 ; 5827 ; 5890$; $5971 ; 6612 ; 7020 ; 9458$.

$\mathrm{MACN}=1188 ; 5196 ; 9391 ; 9606 ; 10261 ; 16324 ; 16883 ; 16986 ; 17320 ; 18216 ; 19173 ; 19262$; $19381 ; 20188 ; 20194 ; 25941 ; \mathrm{S} / \mathrm{N}^{\mathrm{o}}=1$. 
CUADRO 1 (Continuación...)

Material examinado en las distintas colecciones: MLP (Museo de La Plata); MACN

(Museo Argentino de Ciencias Naturales); FML (Fundación Miguel Lillo)

TABLE 1 (Continued...)

Material examined in the different collections: (Museo de La Plata); MACN

(Museo Argentino de Ciencias Naturales); FML (Fundación Miguel Lillo)

Especie

M. soleniformis

Corbiculidae

C. fluminea

C. largillierti

N. limosa

N. paranensis

Solecurtidae

T. gibbus

Sphaeriidae

B. paranensis

E. elliptica

E. guaraniana

E. iguazuensis

E. klappenbachi

E. platensis

M. argentinum

M. patagonicum

P. chicha

P. chiquitanum

P. inacayali

P. magallanicum
Números de Colección

$\mathrm{MLP}=5799 ; 5836 ; 5889 ; 5979 ; 5980 ; 6640 ; 6683$.

$\mathrm{MACN}=5348 ; 6340 ; 6369 ; 6428 ; 8761 ; 8771 ; 9043 ; 14713 ; 19187$.

$\mathrm{MLP}=4700 ; 5100 ; 5141 ; 5152 ; 5264 ; 5323 ; 5329 ; 5330 ; 5332 ; 5336 ; 5386 ; 5406 ; 5410 ; 5413$; $5430 ; 5432 ; 5437 ; 5438 ; 5441 ; 5445 ; 5447 ; 5450 ; 5451 ; 5454 ; 5456 ; 5458 ; 5460 ; 5461 ; 5463$; $5466 ; 5469 ; 5471 ; 5476 ; 5477 ; 5479 ; 5526 ; 5646 ; 5652 ; 5656 ; 5669 ; 5670 ; 5671 ; 5674$ al 5677; 5684; 6842; 6845; 6888; 6997; 7002; 7020; 7048; 7052; 7060; 7061; 7066; 7068; 7111; 7115; $7117 ; 7214 ; 8395$ al 8397; 8399; 11721; 11804; 11805; 11849 .

$\mathrm{MLP}=4699 ; 5101 ; 5139 ; 5140 ; 5200 ; 5256 ; 5258 ; 5337 ; 5442$ al $5444 ; 5452 ; 5455 ; 5459 ; 5465$; $5467 ; 5468 ; 5472 ; 5473 ; 5475 ; 5478 ; 5662 ; 5672 ; 5685 ; 6844 ; 6889 ; 6894 ; 6904 ; 6988 ; 7055$; $7114 ; 7116 ; 7385$.

$\mathrm{MLP}=1778 ; 1846 ; 2699 ; 4390 ; 5097 ; 5464 ; 5474 ; 5482 ; 5660 ; 9506$ al $9508 ; 11850$.

$\mathrm{MLP}=4477 ; 5331 ; 5795 ; 5881$.

$\mathrm{MLP}=1864 ; 2309 ; 2316 ; 2326 ; 2482 ; 2533 ; 2555 ; 2559 ; 2708 ; 2719 ; 3631 ; 10710 ; 10712 ; 10713$; 50084.

$\mathrm{FML}=378$.

$\mathrm{MLP}=4705 ; 5005 ; 5006 ; 5480 ; 6084 ; 8188$.

MLP= 4991 y 4992 (Paratipos); 4994; 4995; 5133.

MLP= 4999 y 5000 (Paratipos); 5006; 5026.

$\mathrm{MLP}=4993 ; 4995$ al 4997; 5422.

$\mathrm{MLP}=5314 ; 8797 ; 8798$.

$\mathrm{MLP}=5009 ; 5010 ; 5012 ; 5013 ; 5015 ; 5022$ al 5024; 5155; 5291; 5303; 5366; 5487 al 5489; 5491; $5520 ; 6094 ; 7069 ; 7073 ; 8801$ al 8807.

$\mathrm{MLP}=5025 ; 5055 ; 5069$ al 5071; 5073 al 5076; 5092; 5316; 5334; 5346; 5359; 5409; 5493; 5498; $5511 ; 5519 ; 5523 ; 6850 ; 6851 ; 7063 ; 7065 ; 7355 ; 9438$ al 9447; 11757; 11851; 11852.

$\mathrm{MLP}=5053 ; 5056 ; 5057 ; 5077$ al 5080; 5083; 5311; 5427; 5428; 6966; 9450; 9451.

$\mathrm{MLP}=6899$ (Paratipo); 6545; 6550; 7203; 7404.

$\mathrm{MACN}=36363$ (Paratipo).

$\mathrm{FML}=14775$ (Paratipo).

$\mathrm{MLP}=6527 ; 6528 ; 6552 ; 6554 ; 6991 ; 6993 ; 7370$.

$\mathrm{MLP}=5067$ y 5068 (Paratipos); 5084.

$\mathrm{MLP}=5064 ; 5301 ; 9708$ al 9710. 
CUADRO 1 (Continuación...)

Material examinado en las distintas colecciones: MLP (Museo de La Plata); MACN

(Museo Argentino de Ciencias Naturales); FML (Fundación Miguel Lillo)

TABLE 1 (Continued...)

Material examined in the different collections: (Museo de La Plata); MACN

(Museo Argentino de Ciencias Naturales); FML (Fundación Miguel Lillo)

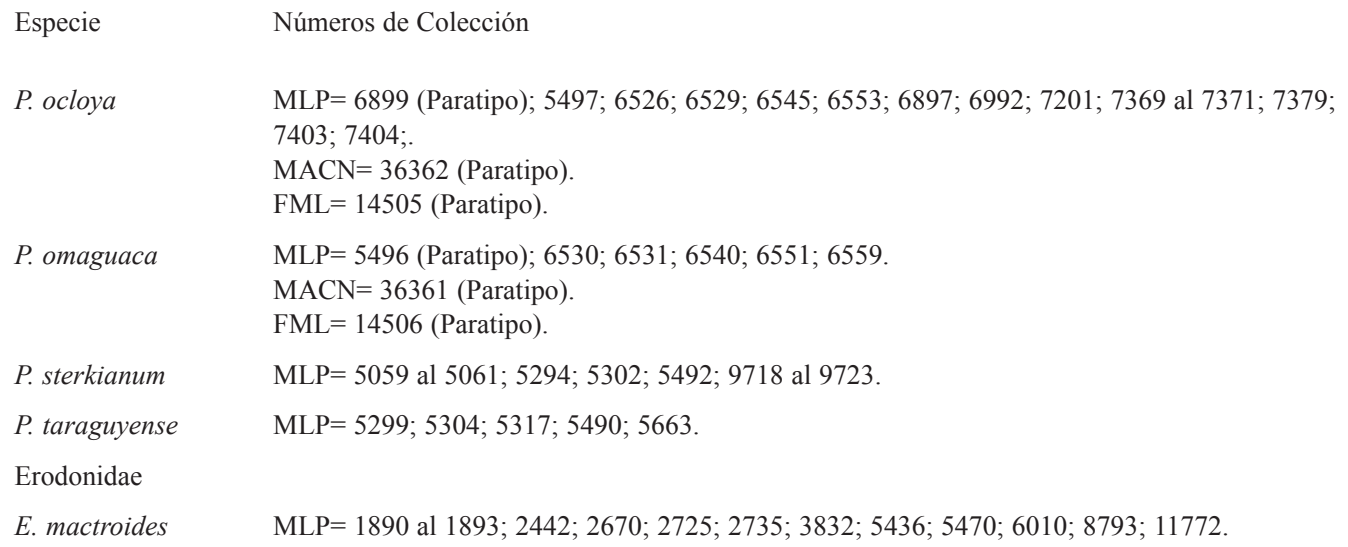

\section{CUADRO 2}

Distribución de especies de moluscos dulceacuícolas por provincias argentinas

TABLE 2

Distribution of species of freshwater molluscs from Argentinean provinces

$\begin{array}{lllllllllllllllllllllllll}\text { / Provincias } & 1 & 2 & 3 & 4 & 5 & 6 & 7 & 8 & 9 & 10 & 11 & 12 & 13 & 14 & 15 & 16 & 17 & 18 & 19 & 20 & 21 & 22 & 23\end{array}$

\section{AMPULLARIIDAE}

\begin{tabular}{|c|c|c|c|c|c|c|c|c|c|c|c|c|c|c|}
\hline A. platae & & & & & & & & $\mathrm{X}$ & & & & & & X \\
\hline A. puelchella & & & & $\mathrm{X}$ & $\mathrm{X}$ & $\mathrm{X}$ & $\mathrm{X}$ & $\mathrm{X}$ & & & & & $\mathrm{X}$ & $\mathrm{X}$ \\
\hline A. spixii & & & & $\mathrm{X}$ & $\mathrm{X}$ & $\mathrm{X}$ & $\mathrm{X}$ & $\mathrm{X}$ & & & & & $\mathrm{X}$ & \\
\hline F. elongata & & & & & & $\mathrm{X}$ & & $\mathrm{X}$ & & & & & & \\
\hline F. iheringi & & & & & & $\mathrm{X}$ & & $\mathrm{X}$ & & & & & & \\
\hline F. neritiniformis & & & & & & $\mathrm{X}$ & & $\mathrm{X}$ & & & & & & $\mathrm{X}$ \\
\hline M. planogyra & & & & & & & $\mathrm{X}$ & $\mathrm{X}$ & & & & & $\mathrm{X}$ & $\mathrm{X}$ \\
\hline P. canaliculata & $\mathrm{X}$ & $\mathrm{X}$ & $\mathrm{X}$ & $\mathrm{X}$ & $\mathrm{X}$ & $\mathrm{X}$ & $\mathrm{X}$ & $\mathrm{X}$ & $\mathrm{X}$ & $\mathrm{X}$ & $\mathrm{X}$ & $\mathrm{X} \quad \mathrm{X}$ & $\mathrm{X}$ & $\mathrm{X}$ \\
\hline P. insularum & & & & & $\mathrm{X}$ & $\mathrm{X}$ & & $\mathrm{X}$ & & & & & $\mathrm{X}$ & $\mathrm{X}$ \\
\hline P. scalaris & & & & $\mathrm{X}$ & $\mathrm{X}$ & & $\mathrm{X}$ & $\mathrm{X}$ & & & & & $\mathrm{X}$ & $\mathrm{X}$ \\
\hline P. americanista & & & & $\mathrm{X}$ & & $\mathrm{X}$ & $\mathrm{X}$ & & & & & & & \\
\hline P. megastoma & & & & & & $\mathrm{X}$ & $\mathrm{X}$ & $\mathrm{X}$ & & & & & & $\mathrm{X}$ \\
\hline
\end{tabular}


CUADRO 2 (Continuación...)

Distribución de especies de moluscos dulceacuícolas por provincias argentinas

TABLE 2 (Continued...)

Distribution of species of freshwater molluscs from Argentinean provinces

/ Provincias

$\begin{array}{llll}1 & 2 & 3 & 4\end{array}$

56

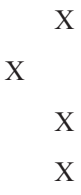

COCHLIOPIDAE

H. australis

H. castellanosae

H. conexa

H. guaranitica

H. hatcheri

H. isabelleana

H. kuesteri

H. montana

H. occidentalis

H. parchappii

H. peiranoi

H. piscium

H. rionegrina

H. sublineata

H. tucumana

H. vianai

LITHOGLYPHIDAE

P. agapetus

P. buschii

P. callosus

P. catharinae

P. concordianus

P. conicus

P. dinochilus

P. doeringi

P. hidalgoi

P. iheringi

X

$\mathrm{X}$

X

X

X
X $\quad X \quad X \quad X$

X

X

$\mathrm{X} \quad \mathrm{X}$

$\mathrm{X} X \mathrm{X} \times \mathrm{X}$

$\mathrm{X}$

X

X

X X X

X X

X X

X

X

X

X

X

X

X X

X

X

X X

X
X X X

$\mathrm{X}$

X

X

X

X

X

X X X 
CUADRO 2 (Continuación...)

Distribución de especies de moluscos dulceacuícolas por provincias argentinas

TABLE 2 (Continued...)

Distribution of species of freshwater molluscs from Argentinean provinces

\begin{tabular}{|c|c|c|c|c|c|c|c|c|c|c|c|c|c|c|c|c|c|c|c|c|c|}
\hline / Provincias & 1 & 2 & 3 & 45 & 6 & 7 & 8 & 9 & 10 & $11 \quad 12$ & 13 & 14 & 15 & 16 & 17 & 18 & 19 & 20 & 21 & 22 & 23 \\
\hline P. lapidum & & & & & $\mathrm{X}$ & $\mathrm{X}$ & $\mathrm{X}$ & & & & & & & & & $\mathrm{X}$ & & & & & \\
\hline P. microthauma & & & & & $\mathrm{X}$ & & $\mathrm{X}$ & & & & & & & & & & & & & & \\
\hline P. orbignyi & & & & & & & $\mathrm{X}$ & & & & & & & & & $\mathrm{X}$ & & & & & \\
\hline P. paranensis & & & & & $\mathrm{X}$ & $\mathrm{X}$ & & & & & & & & & & & & & & & \\
\hline P. peristomatus & & & & & $\mathrm{X}$ & $X$ & & & & & & & & & & & & & & & \\
\hline P. petitianus & & & & & $\mathrm{X}$ & $\mathrm{X}$ & $\mathrm{X}$ & & & & & & & & & $\mathrm{X}$ & & & & & \\
\hline P. philipianus & & & & & $\mathrm{X}$ & $\mathrm{X}$ & $\mathrm{X}$ & & & & & & & & & & & & & & \\
\hline P. quadratus & & & & & & & $\mathrm{X}$ & & & & & & & & & & & & & & \\
\hline P. rushii & & & & & & & $\mathrm{X}$ & & & & & & & & & & & & & & \\
\hline P. simplex & & & & & $\mathrm{X}$ & & $\mathrm{X}$ & & & & & & & & & & & & & & \\
\hline P. tricostatus & & & & & & & $\mathrm{X}$ & & & & & & & & & & & & & & \\
\hline P. valchetensis & & & & & & & & & & & & & & & & & & $\mathrm{X}$ & & & \\
\hline Chilinidae & & & & & & & & & & & & & & & & & & & & & \\
\hline C. aurantia & & & & & & & & & & & & & & & & & $\mathrm{X}$ & & $\mathrm{X}$ & & \\
\hline C. dombeyana & & & & & & & & & & & & & & & & & $\mathrm{X}$ & $\mathrm{X}$ & $\mathrm{X}$ & $\mathrm{X}$ & \\
\hline C. fluminea & & & $X$ & & & & & & & & & & $\mathrm{X}$ & & & $\mathrm{X}$ & & & & & \\
\hline C. fulgurata & & & & & & & & & & & & & & & & & $\mathrm{X}$ & & $\mathrm{X}$ & $\mathrm{X}$ & \\
\hline C. gallardoi & & & & & $\mathrm{X}$ & & $\mathrm{X}$ & & & & & & & & & & & & & & \\
\hline C. gibbosa & & & & & & & & & & & & & & & & $\mathrm{X}$ & $\mathrm{X}$ & $\mathrm{X}$ & $\mathrm{X}$ & $\mathrm{X}$ & \\
\hline C. guaraniana & & & & & $\mathrm{X}$ & & & & & & & & & & & & & & & & \\
\hline C. megastoma & & & & & $\mathrm{X}$ & & & & & & & & & & & $\mathrm{X}$ & & & & & \\
\hline C. mendozana & & & & & & & & & $\mathrm{X}$ & & & $\mathrm{X}$ & & & & & & & & & \\
\hline C. neuquenensis & & & & & & & & & & & & & & & & & $\mathrm{X}$ & $\mathrm{X}$ & $\mathrm{X}$ & & \\
\hline C. parchappii & & $\mathrm{X}$ & & & & & & & & & & $\mathrm{X}$ & & & $\mathrm{X}$ & $\mathrm{X}$ & & $\mathrm{X}$ & & & \\
\hline C. patagonica & & & & & & & & & & & & & & & & & & $\mathrm{X}$ & & $\mathrm{X}$ & $\mathrm{X}$ \\
\hline C. perrieri & & & & & & & & & & & & & & & & & & & & $\mathrm{X}$ & \\
\hline C. portillensis & $\mathrm{X}$ & $\mathrm{X}$ & & & & & & & & & & & & & & & & & & & \\
\hline C. strebeli & & & & & & & & & & & & & & & & & & & & $\mathrm{X}$ & \\
\hline C. rushii & & & & & $\mathrm{X}$ & & $\mathrm{X}$ & & & & & & & & & $\mathrm{X}$ & & & & & \\
\hline \multicolumn{22}{|c|}{ GLACIDORBIDAE } \\
\hline G. magallanicus & & & & & & & & & & & & & & & & & & $\mathrm{X}$ & & & $\mathrm{X}$ \\
\hline \multicolumn{22}{|l|}{ LYMNAEIDAE } \\
\hline “L.” columella & & & & & $\mathrm{X}$ & $\mathrm{X}$ & $\mathrm{X}$ & & & & & & & $\mathrm{X}$ & & $\mathrm{X}$ & & & & & \\
\hline L. diaphana & & & & & & & & & & & & & & & & & $\mathrm{X}$ & & $\mathrm{X}$ & $\mathrm{X}$ & $\mathrm{X}$ \\
\hline
\end{tabular}


CUADRO 2 (Continuación...)

Distribución de especies de moluscos dulceacuícolas por provincias argentinas

TABLE 2 (Continued...)

Distribution of species of freshwater molluscs from Argentinean provinces

/ Provincias

$\begin{array}{lllll}1 & 2 & 3 & 4 & 5\end{array}$

L. pictonica

$\begin{array}{lllllllllllllll}9 & 10 & 11 & 12 & 13 & 14 & 15 & 16 & 17 & 18 & 19 & 20 & 21 & 22 & 23\end{array}$

L. plicata

L. viatrix

X X X

PLANORBIDAE
A. petricola
A. nordestensis
B. intermedia
B. occidentales
B. oligoza
B. orbignyi
B. peregrina
B. straminea
B. tenagophila
D. anatinum
D. cimex
D. depressissimun
D. heloicum
D. kermatoides
D. lucidum

ANCYLIDAE

A. obliquus

G. ticaga

H. moricandi

$U$. concentricus

\section{PHYSIDAE}

P. aspii

P. loosi

"P. cubensis"

“P.” venustula

"S. marmorata" MYTILIDAE
L. fortunei
M. charruana
M. edulis

$\begin{array}{lllll}X & X & X & X & X\end{array}$

$\begin{array}{cccccccc}X & & X & & X & & X & X \\ X & X & X & X & X & X & X & X \\ & X & & X & X & X & X & X \\ X & X & X & X & X & X & X & X \\ & & & X & X & & X & X \\ & & & & X & X & X & X \\ X & X & & X & X & & X & X \\ & & & & & X & X & \\ & & & & & & & \\ & & & X & X & X & X & X \\ & X & & & X & X & X & X\end{array}$

$\mathrm{X}$ $\begin{array}{lll}X & X & X\end{array}$

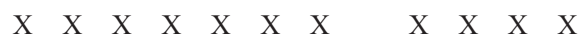
$X \quad X \quad X$ $\mathrm{X} \quad \mathrm{X}$ X $X \quad X$

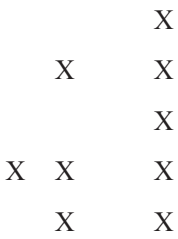

X X
X X

$\mathrm{X}$

$\begin{array}{lllllllllllllllll} & X & X & X\end{array}$ 
CUADRO 2 (Continuación...)

Distribución de especies de moluscos dulceacuicolas por provincias argentinas

TABLE 2 (Continued...)

Distribution of species of freshwater molluscs from Argentinean provinces

/ Provincias

123

6

78

$9 \quad 10$

$\begin{array}{lllllllll}15 & 16 & 17 & 18 & 19 & 20 & 21 & 22 & 23\end{array}$

HYRIIDAE

Castalia ambigua

C. psammoica

D. chilensis

D. delodontus

D. granosus

D. parallelopipedon

D. parodizi

D. rhuacoicus

D. rotundus

D. trifidus

D. charruanus

D. hylaeus

D. variabilis

ETHERIIDAE
A. obtusus
A. tenebricosus
A. trapesialis
A. trapezeus
A. trigonus
A. ensiformis
F. fossiculifera
L. blainvilliana
M. corrientesensis
M. minuana
M. paraguayana
M. parchappii
M. legumen
M. siliquosa
M. soleniformis

CORBICULIDAE

$$
\text { X }
$$$$
\text { X }
$$$$
\text { X X X }
$$$$
\text { X X }
$$$$
\text { X }
$$

X

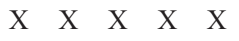

$\begin{array}{llllllllllllll}X & X & X\end{array}$

$\mathrm{X}$

X

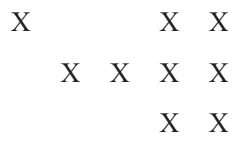

X X

$\mathrm{X} \quad \mathrm{X}$

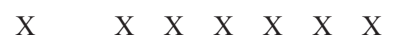

$X \quad X \quad X$

X

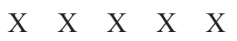

$\mathrm{X} \quad \mathrm{X}$

X X

X X

$\mathrm{X} X \mathrm{X}$

$\mathrm{X} \quad \mathrm{X} \quad \mathrm{X}$

$X \quad X \quad X$

X X

$\mathrm{X}$

$$
\begin{array}{lll} 
& X & X \\
X & X & X \\
X & X
\end{array}
$$

$$
\begin{aligned}
& \text { X X } \\
& \text { X X }
\end{aligned}
$$

X

X X

$\mathrm{X} \quad \mathrm{X}$

$\mathrm{X}$
C. fluminea
$\mathrm{X} \quad \mathrm{X} \quad \mathrm{X} \quad \mathrm{X}$

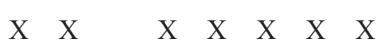
$X \quad X \quad X$
$\mathrm{X}$
$\mathrm{X} \quad \mathrm{X} \quad \mathrm{X}$ 
CUADRO 2 (Continuación...)

Distribución de especies de moluscos dulceacuícolas por provincias argentinas

TABLE 2 (Continued...)

Distribution of species of freshwater molluscs from Argentinean provinces

/ Provincias

N. paranensis

$$
\text { X }
$$

\section{SOLECURTIDAE}

Tagelus gibbus

$$
\text { X X }
$$

\begin{tabular}{|c|c|c|c|}
\hline B. paranensis & & $\mathrm{X}$ & $\mathrm{X}$ \\
\hline E. doellojuradoi & & & $\mathrm{X}$ \\
\hline E. elliptica & & $\mathrm{X}$ & \\
\hline E. guaraniana & & & \\
\hline E. iguazuensis & & $\mathrm{X}$ & \\
\hline E. klappenbachi & & & $\mathrm{X}$ \\
\hline E. platensis & $\mathrm{X}$ & & $\mathrm{X}$ \\
\hline M. argentinum & & $\mathrm{X}$ & $\mathrm{X}$ \\
\hline
\end{tabular}

SPHAERIIDAE

M. patagonicum

P. chicha

P. chilense

P. chiquitanum

P. dorbignyi

P. inacayali

P. magellanicum

P. observationis

P. ocloya

P. omaguaca

P. patagonicum

P. pipoense

P. plenilunium

P. sterkianum

P. taraguyense

P. vile

S. lauricochae

Erodonidae

\begin{tabular}{|c|c|c|c|c|}
\hline$X$ & X & & & \\
\hline$X$ & $\mathrm{X}$ & $X$ & $X$ & $\mathrm{X}$ \\
\hline$X$ & & $X$ & $X \quad X$ & $\mathrm{X}$ \\
\hline
\end{tabular}

E. mactroides
X

$$
\text { X }
$$

X

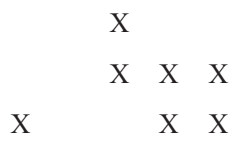

X

X X

X

$\begin{array}{llllllll} & X & X & X & X & X & X & X \\ X & X & & X & & & \\ & & X & & & & & \end{array}$

1: Jujuy; 2: Salta; 3: Tucumán; 4: Formosa; 5: Chaco; 6: Misiones; 7: Corrientes; 8: Entre Ríos; 9: Catamarca; 10: San Juan; 11: La Rioja; 12: San Luis; 13: Santiago del estero; 14: Mendoza; 15: Córdoba; 16: Santa Fe; 17: La Pampa; 18: Buenos Aires; 19: Neuquén; 20: Río Negro; 21: Chubut; 22: Santa Cruz; 23: Tierra del Fuego. 

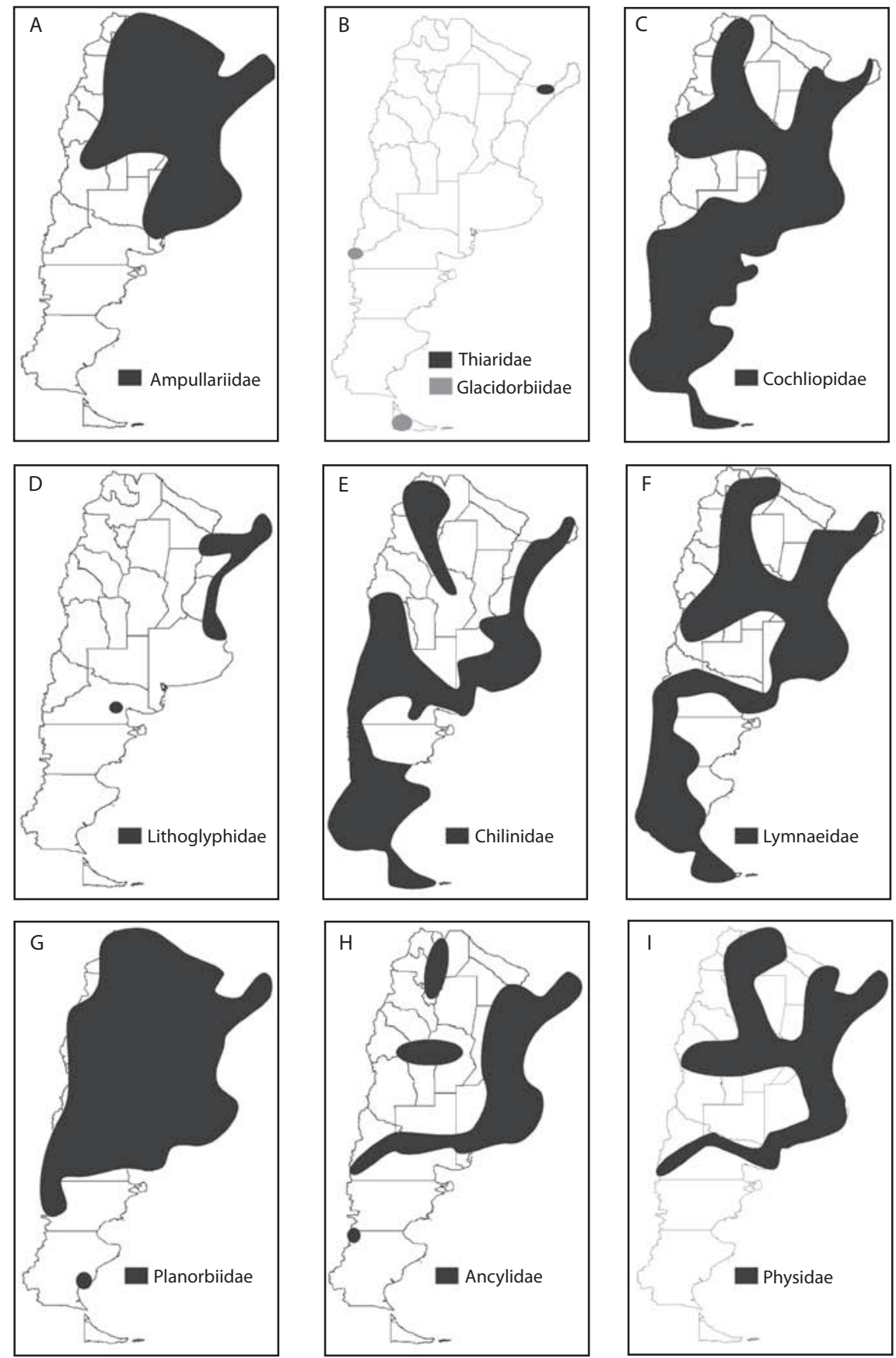

Fig. 2. Distribución de cada familia de Gastropoda dulceacuícolas en la República Argentina.

Fig. 2. Distribution of each family of freshwater Gastropoda in Argentina Republic. 
(Tasmania, Nueva Zelandia, Sudeste y Sudoeste de Australia y Chile), con veinte especies descritas y ordenadas en cinco géneros. Sólo una de ellas está presente en Sudamérica, G. magallanicus, con una distribución discontinua y puntual en Chile, donde radica su localidad tipo, y en la Argentina, con sólo dos registros para Patagonia (lagos Gutiérrez y Yehuín, para las provincias de Río Negro y Tierra del Fuego, respectivamente) (Landoni et al. 1999).

- Chilinidae (Fig. 2E). Esta familia autóctona del sur de Sudamérica, presenta once especies endémicas de la Argentina: $C$. fulgurata, C. gallardoi, C. mendozana, $C$. neuquenensis, C. parchappii, C. portillensis, C. rushii, C. perrieri, C. guaraniana, $C$. megastoma y $C$. strebeli. Las últimas cuatro presentan registros puntuales. Las especies de más amplia distribución son: $C$. gibbosa en el sur (Patagonia), C. parchappii en el centro y $C$. fluminea en el nordeste.

- Lymnaeidae (Fig. 2F). Lymnaea plicata es endémica y presenta una distribución restringida a su localidad típo: Alto río Senguer, provincia de Chubut. Lymnaea diaphana se encuentra mayormente distribuida en toda la Patagonia, mientras que $L$. pictonica se halla solo en el extremo sur de la misma. Lymnaea viatrix se distribuye en el centro y noroeste del país. Por su parte, “ $L$." columella está registrada para el nordeste, en la Mesopotamia.

- Planorbidae (Fig. 2G). Acrorbis petricola está restringida a grandes saltos de cursos de agua que atraviesan la selva misionera. Biomphalaria peregrina es la especie de más amplia distribución en el país. Es necesario aclarar que, a pesar de que $B$. tenagophila está distribuida principalmente en el norte y el centro, existe un registro dudoso para la provincia de Santa Cruz (MLP: 2886), y que consiste de conchillas solamente.

- Ancylidae (Fig. 2H). De las cinco especies registradas para la Argentina, las de distribución más restringida son: A. obliquus, con varios registros para la provincia de Córdoba y uno para la provincia de Río Negro, y Laevapex sp. y G. ticaga, que solamente cuentan con un registro de colección, resultado de colectas propias, para la provincia de Misiones. Sin embargo, Barbosa dos Santos (2003) ya menciona a G. ticaga para la Argentina, sin especificar la localidad o procedencia de la cita.

- Physidae (Fig. 2I). Esta familia presenta pocas entidades descritas para la Argentina. De las cuales "S. marmorata" y "P. cubensis", son las más frecuentes pobladoras de los ambientes dulceacuícolas. Physa aspii y $P$. loosi serían las únicas especies endémicas para la Argentina.

- Mytilidae (Fig. 3A). De las tres especies presentes en aguas continentales, dos de ellas (M. charruana y M. edulis) son marinas a mixohalinas pudiendose encontrar en el estuario del Río de la Plata. En este trabajo solo se tomaron en cuenta los lotes de ambientes mixohalinos de estas especies. La especie restante, L. fortunei es dulceacuícola e introducida en Argentina (ver item especies introducidas).

- Hyriidae (Fig. 3B). Toda la familia se encuentra en el sector NE de la Argentina, salvo $D$. (D.) chilensis que se distribuye en la patagonia Argentina (provincias de Río Negro, Neuquén y Chubut). La única especie con registros puntuales en el NE es D. (D.) granosus.

- Etheriidae (Fig. 3C). Anodontites (A.) puelchanus es la única especie de esta familia que se distribuye en el Sur Argentino, en los ríos Limay y Negro y es endémica de esta cuenca. El resto de las especies se distribuyen en el NE Argentino, por los ríos Uruguay, Paraná, Paraguay, Río de la Plata y sus afluentes. Todas las especies poseen varios registros.

- Corbiculidae (Fig. 3D). Dos de las cuatro especies presentes en el país, son autóctonas: $N$. limosa y $N$. paranensis y se distribuyen por todo el NE argentino. Sin 

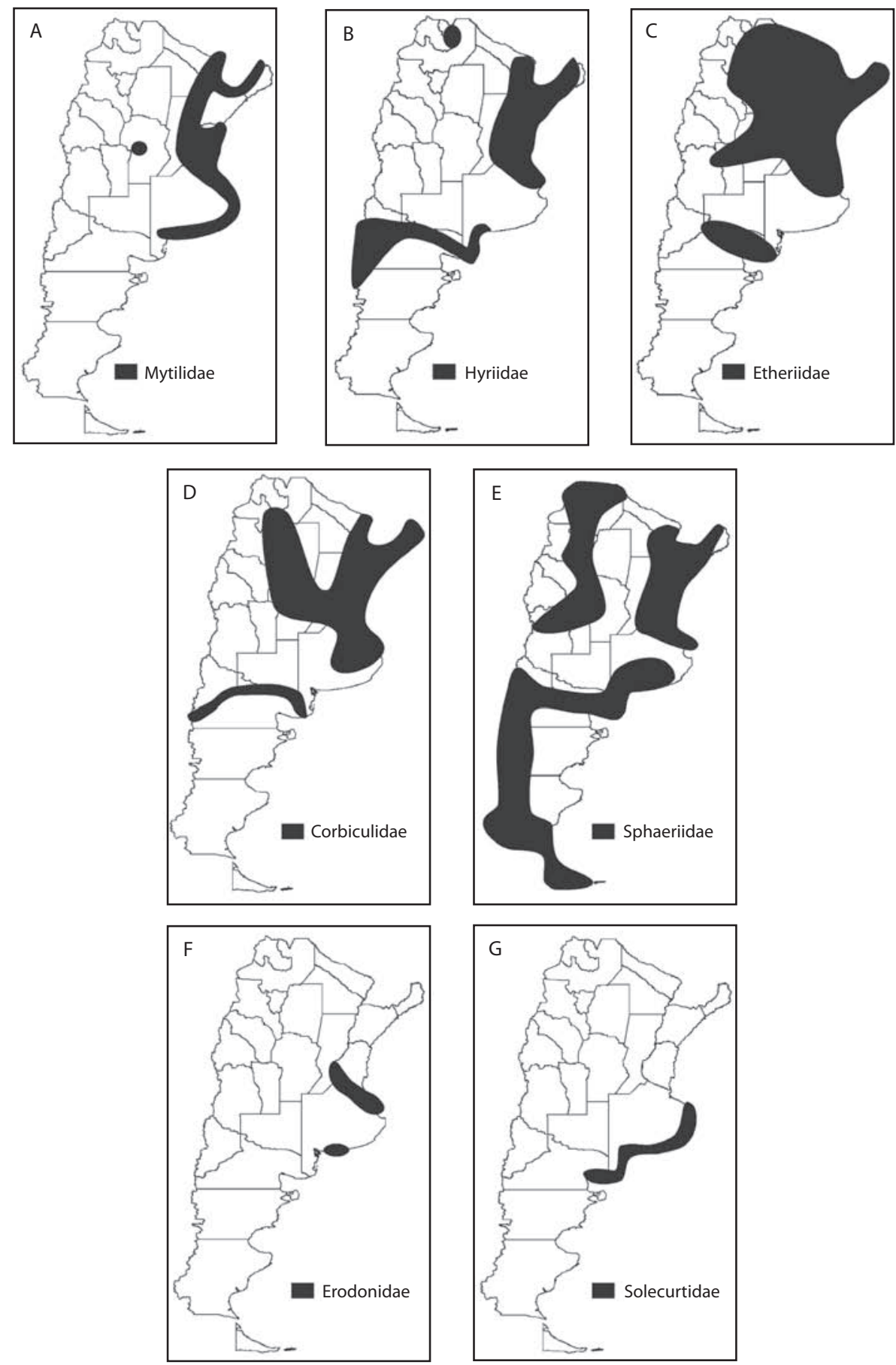

Fig. 3. Distribución de cada familia de Bivalvos dulceacuícolas en la República Argentina.

Fig. 3. Distribution of each family of freshwater Bivalvia in Argentina Republic. 
embargo, la introducción de dos especies asiáticas (C. fluminea y C. largillerti) está ejerciendo una presión de desplazamiento sobre ellas (ver ítem especies introducidas) (Ituarte 1994).

- Sphaeriidae (Fig. 3E). La mayoría de las especies se encuentran en el NE (incluídas todas las del género Eupera). Muy pocas especies se distribuyen por ríos y lagos de los Andes patagónicos. Eupera elliptica, E. guaraniana, E. iguazuensis, $P$. chilensis, $P$. pipoense y $P$. sterkianum poseen un solo registro en Argentina. Se pueden mencionar como endémicas a: $B$. paranesis, $E$. elliptica, E. guaraniana, E. iguazuensis, $P$. chicha, P. inacayali, P. ocloya, P. omaguaca, $P$. patagonicum y $P$. pipoense.

- Erodonidae y Solecurtidae (Fig. 3F y 3G, respectivamente). Familias de abolengo marino que poseen respectivamente una especie con hábitos mixo a eurihalinos, que suelen ser encontradas en estuarios y desembocaduras de ríos en el Mar Argentino a lo largo de la costa de la Provincia de Buenos Aires.

Moluscos de interés sanitario: las especies de Gastropoda de agua dulce son frecuentes hospederos intermediarios de fauna parásita que tiene como hospederos definitivos a humanos y otros vertebrados, entre ellos animales domésticos (ganado) y de importancia económica (peces).

- Planorbidae. Especies del género Biomphalaria son propagadoras naturales o potenciales de la esquistosomiasis, parasitosis humana causada por el Digeneo, Schistosoma mansoni Sambon, 1907. Desde su introducción en América desde África, se viene dispersando hacia el sur, por el E y desde el NE de Brasil. Bernardini y Machado (1981) detectan un foco de infección esquistosómica en la localidad de San Francisco Do Sul, Estado de Santa Catarina, Brasil, en las cabeceras del río Iguazú. Graeff-Texeira et al. (1999) identificaron el foco más austral en el Estado de
Río Grande do Sul, en Porto Alegre, Brasil. De las especies naturalmente propagadoras en Brasil encontramos, en Argentina, a $B$. tengophila y $B$. straminea, de amplia distribución y muy frecuentes en áreas de la cuenca del Plata. Biomphalaria peregrina es potencial propagadora de la endemia, ya que su infestación ha sido demostrada en laboratorio, junto a otras especies del género, y aún no ha sido hallada infectada en su medio natural (Rumi 1991). Áreas de mayor riesgo de generación de focos endémicos de esquistosomiasis en la Argentina son aquellas con estrecha relación con los ríos más importantes de la cuenca del Plata en la Argentina: Paraná, Uruguay y Río de la Plata, con gran movilidad de recursos humanos en las áreas limítrofes.

- Lymnaeidae. Caracoles de esta familia son hospederos intermediarios de Fasciola hepatica Linné, 1758 (Trematoda, Digenea, Fasciolidae) productoras de fasciolasis o distomatosis. Esta parasitosis es de distribución cosmopolita y considerada mundialmente una de las enfermedades parasitarias más importantes del ganado, especialmente bovino y ovino. La fasciolasis humana ocurre en forma esporádica $\mathrm{o}$ en brotes y se ha registrado en numerosos países de América, Europa, África y Asia (Acha y Szyfres 1997). "Lymnaea" columella y L. viatrix son hospedadores intermediarios de fasciolasis en Argentina.

- Chilinidae. Especies de esta familia forman parte del grupo de gasterópodos de agua dulce que actúan como hospederos intermediarios de furcocercarias (estadios larvarios) de la familia Schistosomatidae (Trematoda: Digenea), productoras de dermatitis esquistosómicas humanas. Uno de los casos más severos de dermatitis esquistosómica humana, en Argentina, se detectó en el lago Pellegrini ( $38^{\circ} 40^{\prime} \mathrm{S}$ y $\left.68^{\circ} 00^{\prime} \mathrm{W}\right)$, provincia de Río Negro, siendo el hospedador $C$. gibbosa (Martorelli, 1984). En áreas relacionadas al Delta del Paraná, Szidat (1951) y Miquel (1984) detectaron furcocercarias esquistosómicas en Chilina fluminea. 
- Physidae, Coclhiopidae, Ampullariidae. Especies de estas familias son hospederas intermediarias de trematodos de la familia Schistosomatidae, cuyas cercarias producen el mismo tipo de dermatitis descrita para las especies de la familia Chilinidae (Ostrowski de Núñez 1992). Aún no se han detectado focos de dermatitis en los cuales estén involucradas estas especies.

Especies exóticas: son aquellas reubicadas por el hombre y que colonizan nuevas y lejanas regiones respecto de su ámbito de distribución natural. Aunque los movimientos dispersivos de las especies cambian naturalmente a lo largo del tiempo, la actividad del hombre incrementa en gran medida la tasa y escala espacial de estos cambios, ya sea de forma intencional o no. Estas especies son transportadas de distintas formas, por: acuarios (escapes); liberación de carnada por los pescadores deportivos; para conservación de la especie y/o liberación para control biológico; botes y actividad náutica-agua de lastre; canales de riego o navegación y, por último, transporte de comida viva y/o por vía terrestre.

Las características propias de las especies no-nativas o exóticas ("invasiveness"), más las interacciones y características del ambiente a invadir ("invasibility"), determinan como comienzan éstas a distribuirse. Algunas continúan relativamente cerca del punto de introducción (especies no-invasoras), mientras que otras se dispersan ampliamente (especies invasoras).

- Thiaridae. Melanoides tuberculatus es originario del Sur de Asia, Australia y Africa. En la década del '60 se la introduce en el Caribe y más recientemente en Sudamérica (Venezuela, Ecuador, Brasil, Perú). Peso y Quintana (1999) publican el primer registro de esta especie en Argentina para la zona de la represa de Yacyretá, provincia de Corrientes.

- Lymnaeidae. Según Castellanos y Landoni (1981), "Lymnaea" columella es exótica en Argentina.
- Planorbidae. Helisoma duryi (Wetherby 1879) $(\mathrm{MLP}=5650)$, nativa de América del Norte, fue registrada en acuarios de venta de peces, pero no en ambientes naturales aún, de todas maneras es una alerta al riesgo de introducción en Argentina (Rumi et al. 2002).

- Physidae. Miquel y Zelaya (1999) consideran que $P$. cubensis es una especie recientemente introducida, asociada a la importación y transporte de plantas acuáticas; mientras que, los escasos hallazgos de $P$. venustula, no permiten extraer una conclusión acerca de su carácter nativo o exótico.

- Mytilidae. Limnoperna fortunei es un bivalvo de origen asiático que en la década del ' 90 fue detectada por primera vez en el Río de la Plata (Pastorino et al. 1993). Tiene la particularidad de fijarse, por medio del órgano denominado biso, a los sustratos duros. Los ecosistemas acuáticos locales le han sido sumamente favorables para su reproducción y expansión, especialmente la cuenca del río Paraná por la cual ha ascendido rápidamente hasta la represa hidroeléctrica de Itaipú, Brasil. Por el río Uruguay su límite norte lo ha alcanzado en la localidad de Federación, Entre Ríos, en el año 2000 (Darrigran 2002). La capacidad de proliferación, aglomeramiento y fuerte fijación a los sustratos duros (macrofouling) de L. fortunei, está creando problemas muy serios en las tomas de agua instaladas en el Río de la Plata y en represas hidroeléctricas como Yacyretá, Itaipú, Salto Grande, provocando taponamientos en tuberías de captación. Los costosos daños que esta especie invasora produce son similares a los producidos por Dreissena polimorpha (Pallas, 1754) en el hemisferio norte. No solamente es importante su impacto económico sino el que opera sobre la fauna local, provocando reestructuraciones y reemplazos importantes en sus especies. 
- Corbiculidae. Si bien dos especies del género Corbicula, provenientes del sudeste de Asia, ingresaron a fines de los '60 -principios de los '70 a América del Sur por el Río de la Plata $(C$. largillierti y $C$. fluminea), solo una de estas se comportó como especie invasora. Corbicula fluminea o "almeja asiática", desplazó a la cogenérica introducida (Darrigran 1992) y se extendió por gran parte del sub-continente americano, invadiendo a los países que comparten la cuenca del Plata, tal como Brasil, Uruguay, Paraguay y Argentina. En este último, llega hasta el límite norte de la región patagónica (Cazzaniga 1997). En la década de los '80, aparece en los ríos Caripe y San Juan, Venezuela (McMahon 2000). Hay dos hipótesis de introducción de esta especie invasora. Una, a través del sedimento depositado en los tanques de agua de lastre de las embarcaciones transoceánicas. La otra, transportada como alimento de inmigrantes asiáticos a la región (Darrigran 2002). Si bien esta especie es "plaga" por los casos de macrofouling que ocasiona desde su introducción en la década del '30 en América del Norte, en América del Sur, solo desde el año 2000, se detectan problemas en las plantas generadoras de energía de Brasil (Zampatti y Darrigran 2001). Asimismo, si bien no existen estudios en América del Sur sobre impacto ocasionado por C. fluminea en la fauna nativa, cabe esperar que las altas densidades que puede desarrollar en los ambientes invadidos (aproximadamente 1 $500 \mathrm{ind} / \mathrm{m}^{2}$ según Darrigran 1991), impacta sobre el bentos nativo, como así también en la dieta de potenciales depredadores, como los peces (Darrigran y Colauti 1994).

Acciones recomendadas para la conservación de la biodiversidad: la biodiversidad está siendo desbastada por acción del hombre a través de la destrucción de sus hábitats y por la introducción de especies no-nativas (o contaminación por especies). Es prioritaria la formación de nuevas áreas protegidas, con la finalidad de preservar las biotas particulares del territorio y el ambiente en general. Es necesario prestar especial atención a las especies de moluscos dulceacuícolas endémicas presentes en las áreas que actualmente sufren mayor presión urbana, contaminación de cuerpos de agua y/o degradación por deforestación, como lo es la región Mesopotámica de la Argentina.

En la década de los '90, se generó el marco legislativo necesario para instrumentar una gestión ambiental adecuada en la Argentina. El artículo 41 de la nueva Constitución Nacional de la República Argentina, explica, entre otros puntos relativos al ambiente, la "preservación de la diversidad biológica". A tal efecto, los problemas a resolver pueden generalizarse en los siguientes tres puntos:

1. Evaluar el estado actual de la diversidad específica, genética y ambiental;

2. Conocer los problemas que enfrenta la biodiversidad del territorio y establecer un orden de prioridades para solucionarlos;

3. Concientizar a la población sobre la necesidad de un desarrollo sustentable, para lo cual se debe proteger y conservar la biodiversidad del territorio. El desarrollo de los tres puntos anteriores, involucra una serie de etapas, donde la investigación científica es la generadora del conocimiento básico, la cual proporcionará las herramientas necesarias para definir las dos etapas siguientes: a- Toma de decisiones en el marco de un desarrollo sustentable, que abarca espacios de manejo, gestión o administración. b- Educación ambiental.

\section{AGRADECIMIENTOS}

Se agradece especialmente a los siguientes especialistas, miembros de la Facultad de Ciencias Naturales y Museo de la Universidad Nacional de La Plata (FCN y M-UNLP), Argentina, que han colaborado en la identificación del material: M.F. López Armengol (Lithoglyphidae), S.M. Martín (Cochliopidae y Ampullariidae), M.P. Tassara e I.I. César 
(Ancylidae, Hyriidae y Etheriidae), M.A. Roche (Lymnaeidae) y a C. Ituarte (Sphaeriidae). También agradecemos a S. Barbosa dos Santos, FIOCRUZ, Río de Janeiro, Brasil, la identificación de material de la provincia de Misiones. Este trabajo fue subsidiado por CONICET (PIP $\mathrm{N}^{\circ} 2711$ y PIP $\mathrm{N}^{\circ}$ 6370), BID 1728/OC-ARPICT 25621 y por la FCNyM-UNLP (Proyecto Programa Incentivos $N^{\circ} 11 / 352$ ).

\section{RESUMEN}

Se presenta un estado de situación y una lista actualizada y comentada sobre las especies de moluscos de agua dulce de la República Argentina. Se aborda en Gastropoda y Bivalvia la distribución a nivel de familias; las entidades endémicas, exóticas, invasoras y de importancia sanitaria. Los moluscos relacionados a la cuenca del Plata presentan la mayor riqueza específica. Base de datos: 4500 registros relevados de las tres colecciones más importantes de la Argentina: MLP, MACN y FML. Además, se incluye información de recolecciones actuales y localidades citadas por otros autores. Hasta el presente han sido descritas 166 especies. De ellas 101 pertenecen a 10 familias de Gastropoda y 65 a 7 de Bivalvia. Las familias que presentan mayor riqueza específica son Lithoglyphidae (22) y Sphaeriidae (25), respectivamente. Sphaeriidae, Cochliopidae, Chilinidae y Lymnaeidae se distribuyen prácticamente en todo el país. Erodonidae y Solecurtidae se registran en ambientes mixohalinos de la provincia de Buenos Aires. Thiaridae y Glacidorbiidae presentan una distribución muy restringida. Especies endémicas de la Argentina: Gastropoda: Ampullariidae (1), Cochliopidae (10), Lithoglyphidae (11), Thiariidae (3), Chilinidae (11), Lymnaeidae (2) y Physidae (2?); Bivalvia: Hyriidae (1?); Etheriidae (1?) y Sphaeriidae (10). Especies de interés sanitario: propagadoras de: Esquistosomiasis, Biomphalaria peregrina, B. straminea y B. tenagophila (Planorbidae); Fasciolasis, Lymnaea viatrix y L. columnella (Lymnaeidae); y dermatitis esquistosómicas, Chilina gibbosa y C. fluminea (Chilinidae). Especies de origen asiático: Corbicula fluminea (Corbiculidae) y Limnoperna fortunei (Mytilidae). Es prioritaria la formación de áreas protegidas para la conservación de especies endémicas, especialmente de la Mesopotamia Argentina.

Palabras clave: moluscos de agua dulce, Gastropoda, Bivalvia, Argentina.

\section{REFERENCIAS}

Acha, P.N. \& B. Szyfres. 1997. Zoonosis y enfermedades transmisibles comunes al hombre y a los animales. OPS, Washington, EEUU.
Anónimo. 1989. Names tour countries with major illegal wildlife trafficking problems. World Wildlife Fund (WWF), Press Release, Gland.

Anónimo. 1998. Freshwater Biodiversity: A preliminary Global Assessment. Draft circulated at the Fourth Meeting of the Conference of the Parties to the Convention on Biological Diversity. World Conservation Monitoring Centre (WCMC), Bratislava, Eslovaquia.

Anónimo. 2000. Gasoducto Norandino: parte de la solución. Rev. Fund. Vida Silv. 72: 4-15.

Barbosa dos Santos, S. 2003. Estado actual do conhecimento dos ancilídeos na América do Sul (Mollusca: Gastropoda: Pulmonata: Basommatophora). Rev. Biol. Trop. 51: 191-224.

Barrientos, Z. \& J. Monge-Nájera. 2003. Malacologia Latinoamericana. Rev. Biol. Trop. 51: 1-536.

Bernardini, O. \& M. Machado. 1981. Esquistossomose mansoni em Santa Catarina: isolamento do Schistosoma mansoni do primeiro foco de transmissao ativa em Sao Francisco do Sul. Nota previa. Arq. Catarinenses Med. 10: 213.

Bertonatti, C. \& J. Corcuera. 2000. Situación Ambiental Argentina. 2000. Fundación Vida Silvestre Argentina, Buenos Aires, Argentina.

Bonetto, A. 1961. Acerca de la distribución geográfica de los Náyades de la República Argentina. Physis 22: 259-268.

Bonetto, A. 1962. Especies del género Mycetopoda en el sistema hidrográfico del río de la Plata. Rev. Mus. Arg. Cien. Natur. Bernardino Rivadavia 8: 173-182.

Bonetto, A. 1963. Contribución al conocimiento de Leila blainvilleana (Lea) (Mollusca: Pelecypoda). Physis 24: 11-16.

Bonetto,A.1966.EspeciesdelasubfamiliaMonocondylaeinae en las aguas del Río de la Plata (Mollusca: Mutelacea). Arch. Molluskenk 95: 3-14.

Bonetto, A. 1967. El género Anodontites Bruguiere (Mollusca, Pelecipoda) en el sistema hidrográfico del Plata. Physis 26: 459-467.

Bonetto, A. 1973. Náyades de la Patagonia. Rev. Asoc. Cienc. Natur. Litoral 4: 177-185.

Bonetto, A., J.A. Bechara \& M. Tassara. 1982. Los moluscos de la familia Planorbidae en el área del río Paraná Medio. Physis B 41: 1-6. 
Bonetto, A. \& I. Ezcurra. 1965. Estudios comparados de las formas larvales de Mutelidae Ortemann y su significación sistemática zoogeográfica (Mollusca, Pelecipoda). II Congreso Latinoamericano Zoología, San Pablo, Brasil.

Bonetto, A. \& M. Tassara. 1987. Contribución al conocimiento de dos náyades sudamericanas. Rev. Mus. Arg. Cien. Natur. Bernardino Rivadavia 14: 163-170.

Bonetto, A. \& M. Tassara. 1988. Contribución al conocimiento limnológico de moluscos Pelecípodos en la cuenca del Plata, con particular referencia a sus relaciones tróficas. Ecosur 14/15: 17-54.

Brown, A.D. \& H.R. Grau. 1993. La Naturaleza y el Hombre en las Selvas de Montaña. Proyecto GTZ. Desarrollo Agroforestal Comunidades Rurales Noroeste Argentino, Salta, Argentina.

Bucher, E.H. \& J.M. Chain. 1998. Capítulo 10. Chaco, p 73-96. In E. Bucher Canevari, D. Blanco, G. Castro \& I. Davidson (eds.). Los humedales de la Argentina: clasificación, situación actual, conservación y legislación. Wetlands International, Publ. No46 (2 ${ }^{\text {da }}$ ed.), Buenos Aires, Argentina.

Bouchet, P. \& J.P. Rocroi. 2005. Classification and nomenclator of Gastropod families. Malacologia 47: 1-397.

Carcelles, A. 1941. Erodona mactroides en el Río de la Plata. Physis 19: 11-21.

de Castellanos, Z.A. 1959. Algunas consideraciones sobre el género Diplodon (Moluscos-Pelecipodos). Rev. Mus. La Plata 182: 243-246

de Castellanos, Z.A. 1981. Mollusca, Gasteropoda, Thiaridae. Fauna de Agua Dulce de la República Argentina, PROFADU (CONICET) Buenos Aires 15: 5-18.

de Castellanos, Z.A. \& D. Fernández. 1976. Mollusca, Gasteropoda, Ampullariidae. Fauna de Agua Dulce de la República Argentina, PROFADU (CONICET) Buenos Aires 15: 5-33.

de Castellanos, Z.A. \& M.C. Gaillard. 1981. Mollusca, Gasteropoda, Chilinidae. Fauna de Agua Dulce de la República Argentina, PROFADU (CONICET) Buenos Aires 15: 23-51.

de Castellanos, Z.A. \& N. Landoni. 1981. Mollusca, Gasteropoda, Lymnaeidae. Fauna de Agua Dulce de la República Argentina, PROFADU (CONICET) Buenos Aires 15: 53-82. de Castellanos, Z.A. \& N. Landoni. 1990. La familia Myceopodidae Gray, 1840 en la República Argentina. Fauna de Agua Dulce de la República Argentina, PROFADU (CONICET) Buenos Aires 16: 5-87.

de Castellanos, Z.A. \& S.E. Miquel. 1980. Notas complementarias al género Chilina Gray (Mollusca Pulmonata). Neotrópica 26: 171-178.

Cazzaniga, N.J. 1981a. Notas sobre Hidrobidos Argentinos. III. (Gastropooda, Rissoidea). Strobeliella, un nuevo género de la Patagonia. Neotrópica 27: 3-10.

Cazzaniga, N.J. 1981b. Sobre la identidad de Littoridina vianai Parodiz, 1960 (Gastropoda: Hydrobiidae). Neotrópica 27: 178.

Cazzaniga, N.J. 1997. Asiatic clam, Corbicula fluminea, reaching Patagonia (Argentina). J. Freshwater Ecol. 12: 629-630.

Censo Nacional. 2001. Instituto Nacional de Estadística y Censos, Argentina. www.indec.gov.ar/webcenso/ index.asp.

Cowie, R. \& S. Thiengo. 2003. The apple snails of the Americas (Mollusca: Gastropoda: Ampullariidae: Asolene, Felipponea, Marisa, Pomacea, Pomella): a nomeclatural and type catalog. Malacologia 45: 41-100.

Cuezzo, M.G. \& F. Drahg. 1995. Moluscos depositados en la colección de la Fundación Miguel Lillo: Argentina. Acta Zool. Lilloana 43: 185-203.

Davis, G.M., M. Mazurkiewicz \& M. Mandracchia. 1982. Spurwinkia: Morphology, Systematics, and Ecology of a new genus of North American Marshaland Hydrobiidae (Molusca: Gastropoda). Proc. Acad. Natur. Sci. Philadelphia 134: 143-177.

Darrigran, G.A. 1991. Aspectos Ecológicos de la Malacofauna Litoral del Río de la Plata. República Argentina. Tesis Doctoral $n^{\circ}$ 568, Facultad Ciencias Naturales y Museo, Univ. Nac. La Plata, La Plata, Argentina.

Darrigran, G.A. 1992. Variación temporal y espacial de la distribución de las especies de Corbicula Megerle, 1811 (Bivalvia, Corbiculidae). Neotrópica 38: 59-63.

Darrigran, G.A. 1995. Distribución de tres especies del género Heleobia Stimpson, 1865 (Gastropoda, Hidrobiidae) en el litoral argentino del Río de la Plata y arroyos afluentes. Iheringia 78: 3-8. 
Darrigran, G.A. 2002. Potential impact of filter-feeding invaders on temperate inland freshwater environments. Biol. Invasion 4: 145-156.

Darrigran, G.A. 2004. Moluscos Invasores, en especial Corbicula fluminea (Almeja asiática) y Limnoperna fortunei (Mejillón dorado), de la región Litoral. In F.G. Aceñolaza (ed.). Temas de la Biodiversidad del Litoral fluvial argentino Insugeo. Miscelánea 12: 205-210.

Darrigran, G.A. \& D. Colauti. 1994. Potencial control biológico del molusco invasor Corbicula fluminea (Müller, 1774) en el Río de la Plata. Comun. Soc. Malacol. Uruguay 7: 368-373.

Darrigran, G.A. \& M.F. LópezArmengol. 1998. Composition, structure and distribution of malacofauna living on a hard substrate at the Argentinian shore of Río de la Plata, Argentina. Gayana 62: 79-89.

Fernández, D. 1981a. Mollusca, Gasteropoda, Physidae. Fauna de Agua Dulce de la República Argentina, PROFADU (CONICET), Buenos Aires 15: 83-98.

Fernández, D. 1981b. Mollusca, Gasteropoda, Ancylidae. Fauna de Agua Dulce de la República Argentina, PROFADU (CONICET), Buenos Aires 15: 99-114.

Gaillard, M.C. \& Z.A. de Castellanos. 1976. Mollusca, Gasteropoda, Hydrobiidae. Fauna de Agua Dulce de la República Argentina, PROFADU (CONICET), Buenos Aires 15: 5-40.

Graeff-Teixeira, C., C.B. dos Anjos, V.C. de Oliveira, C.F. Velloso, M.B. da Fonseca, C. Valar, C. Moraes, C.T. Garrido \& R.S. do Amaral. 1999. Identification of a Transmission Focus of Schistosoma mansoni in the Southernmost Brazilian State, Rio Grande do Sul. Mem. Inst. Oswaldo Cruz 94: 9-10.

Hylton Scott, M.I. 1953. Sobre limnaeidae argentinos. Physis 20: 401-408.

Hylton Scott, M.I. 1954. Dos nuevos Melanidos del Alto Paraná (Mollusca, Prosobranchia). Neotrópica 1: $45-48$.

Hylton Scott, M.I. 1960. Nueva familia de Pulmonados Basommatóforos (Mollusca). Neotrópica 6: 65-69.

Hylton Scott, M.I. 1968. Répartition et biogéographie des Mollusques de Patagonie, p. 269-273. In C. Delamare Debouteville \& E. Rapoport (eds.). Biologie de l'Amerique Australe, 4. CNRS-CONICET, Paris, Francia.
Ituarte, C. 1981. Primera noticia acerca de la introducción de pelecípodos asiáticos en el área rioplatense (Mollusca, Corbiculidae). Neotrópica 27: 79-82.

Ituarte, C. 1989. Los géneros Byssanodonta d'Orbigny, 1846 y Eupera Bourguignat, 1854 Bivalvia: Sphaeriidae) en el área rioplatense. Descripción de Eupera iguazuensis n.sp. del río Iguazú, Misiones, Argentina. Neotrópica 35: 53-63.

Ituarte, C. 1994. Corbicula and Neocorbicula (Bivalvia: Corbculidae) in the Paraná, Uruguay, and Río de la Plata Basins. Nautilus 4: 129-135.

Ituarte, C. 1996. Argentine species of Pisidium Pfeiffer, 1821, and Musculium Link, 1807 (Bivalvia: Sphaeriidae). Veliger 39: 189-203.

Ituarte, C. 2005. The Sphaeriidae (Bivalvia) from northwestern Argentina including three new species of Pisidium. Nautilus 119: 93-104.

Kabat, A. 1997. Correct family names for the freshwater "muteloid" bivalves (Unionoida: Etherioidea). Occasional Papers Molluscs 5: 379-392.

Kabat, A. \& R. Hershler. 1993. The prosobranch snail family Hydrobiidae (Gastropoda: Rissooidea): review of classification and supraspecific taxa. Smithsonian Contribution Zool. 547: 1-87.

Landoni, N.A., A. Rumi \& S. Gordillo. 1999. Sobre la presencia de Glacidorbis Iredale, 1943 en un lago de Tierra del Fuego, Argentina (Mollusca: Gastropoda). IV Congreso Latinoamericano Malacología, Coquimbo, Chile.

López Armengol, M.F. 1985. Estudio sistemático y bioecológico del género Potamolithus (Hydrobiidae) utilizando técnicas de taxonomía numérica. Tesis Doctoral $\mathrm{n}^{\mathrm{o}}$ 455, Facultad Ciencias Naturales y Museo, Univ. Nac. La Plata, La Plata, Argentina.

LópezArmengol, M.F. \& G.A. Darrigran. 1998. Distribución del género neotropical Potamolithus Pilsbry \& Rush, 1896 (Gastropoda: Hydrobiidae) en el estuario del Río de la Plata. Iberus 16: 67-74.

Martorelli, S.R. 1984. Sobre una cercaria de la familia Schistosomatidae (Digenea) parásita de Chilina gibbosa Sowerby, 1841 en el Lago Pellegrini, Provincia de Río Negro, República Argentina. Neotrópica 30: 97-106.

McMahon, R. 2000. Invasive characteristics of the freshwater bivalve Corbicula fluminea, p 505-552. In Claudi \& Leach (eds.). Nonindigenous freshwater 
organisms. Vectors, Biology, and Impacts. Lewis. Boca Raton

Miquel, S.E. 1984. Contribución al conocimiento biológico de gasterópodos pulmonados del área rioplatense, con especial referencia a Chilina fluminea (Maton). Tesis doctoral $n^{\circ} 482$, Facultad Ciencias Naturales y Museo, Univ. Nac. La Plata, Argentina.

Miquel, S.E. \& H. Parent. 1996. Moluscos Gasterópodos de la provincia de Santa Fe, Argentina. Malacol. Rev. 29: $107-112$.

Miquel, S.E. \& D.G. Zelaya. 1999. Nuevos hallazgos de Physella cubensis y $P$. venustula en la Argentina (Mollusca, Physidae). Physis B 57: 9-10.

Olazarri, J. 1977. Informe preliminar sobre moluscos del área de influencia de la futura represa de Salto Grande. Reunión Aspectos Desarrollo Ambiental 4, Salto (Uruguay)-Concordia (Argentina) 7: 1-25.

Olazarri, J. 1980. La formación del embalse de Salto Grande y sus efectos sobre la malacofauna fluvial. Resúmenes Jornadas Ciencias Naturales Uruguay, Montevideo, Uruguay.

Ostrowski de Núñez, M. 1992. Trematoda. Familias Strigeidae, Diplostomidae, Schistosomatidae, Spirorchiidae y Bucephalidae. Fauna de Agua Dulce de la República Argentina, PROFADU (CONICET), Buenos Aires 9: 5-55.

Paraense, W.L. 1974. Biomphalaria oligoza n.n. for Tropicorbis philippianus (Dunker) sensu Lucena. Rev. Brasileira Biol. 34: 379-386.

Paraense, W.L. 1983. Lymnaea columella in northern Brazil. Mem. Inst. Oswaldo Cruz 78: 477-482.

Paraense, W.L. 1985. Biomphalaria intermedia in Matto Grosso do Sul, Brazil and Misiones, Argentina (Pulmonata: Planorbidae). Mem. Inst. Oswaldo Cruz 80: $247-250$

Paraense, W.L. \& J.P. Pointier. 2003. Physa acuta Draparnaud, 1805 (Gastropoda: Physidae): a study of topotypic specimens. Mem. Inst. Oswaldo Cruz 98: 513-517.

Parodiz, J.J. 1965. The hydrobid snails of the genus Potamolithus (Mesogastropoda: Rissoacea). Sterkiana 20: 1-38.
Parodiz, J.J. \& L. Hennings. 1965. The Neocorbicula (Mollusca: Pelecypoda) of the Parana-Uruguay basin, South American. Ann. Carnegie Mus. 38: 69-96.

Pastorino, G., G. Darrigran, S. Martín \& L. Lunaschi. 1993. Limnoperna fortunei (Dunker, 1857) (Mytilidae), nuevo bivalvo invasor en aguas del Río de la Plata. Neotrópica 39: 101-102.

Peso, J.G. \& M.G. Quintana. 1999. Otro molusco de origen asiático introducido en la Cuenca del Plata: Melanoides tuberculata en el embalse de Yacyretá, Argentina/Paraguay (Prosobranchiata: Thiaridae). IV Congreso Latinoamericano Malacología, Coquimbo, Chile.

Ponder, W. \& G. Avern. 2000. The Glacidorbidae (Mollusca: Gastropoda: Heterobranchia) of Australia. Rec. Australian Mus. 52: 307-357.

Quintana, M.G. \& A.C. Mercado Laczkó. 1997. Biodiversidad en peligro. Caracoles de los rápidos en Yaciretá. Cienc. Hoy 7: 22-31.

Rumi, A. 1986. Estudio morfológico, taxonómico y bio-ecológico de los planórbidos argentinos. Tesis Doctoral $\mathrm{n}^{\circ}$ 461, Facultad Ciencias Naturales $\mathrm{y}$ Museo, Univ. Nac. La Plata, Argentina.

Rumi, A. 1991. La Familia Planorbidae Rafinesque, 1815 en la República Argentina. Fauna de Agua Dulce de la República Argentina, PROFADU (CONICET), Buenos Aires 15: 3-51.

Rumi, A., A. Paola \& M. Tassara. 2002. Introduction risk of alien species: Helisoma druyi (Wetherby, 1879) (Gastropoda: Planorbidae) in Argentina. Natura Neotropicalis 33: 91-94.

Rumi, A., M. Tassara \& A.A. Bonetto. 1997. Distribución de las especies de Planorbidae en la Argentina y su relación con el riesgo de esquistosomiasis. Ecosur 17: 1-19.

Rudolph, P. 1983. A prostate pouch in Pseudosuccinea columella (Basommatophora: Lymnaeidae). Walkerana 1:389-394.

Scarabino, F. 2004. Lista sistemática de los Gastropoda dulceacuícolas vivientes de Uruguay. Comun. Soc. Malacol. Uruguay 8: 347-356. 
Szidat, L. 1951. Cercarias schistosómicas y dermatitis schistosómica humana en la República Argentina. Com. Inst. Nac. Inv. Cienc. Nat. Cienc. Zool. 2: $129-150$.

Tassara, M.P. \& J.A. Bechara. 1983. Sobre la presencia de Biomphalaria oligoza Paranense, 1974 (Gastropoda, Planorbidae) en la cuenca del Río Paraná Medio. Neotrópica 29: 215-219.

Taylor, D. 2003. Introduction to Physidae (Gastropoda: Hygrophila); biogeography, classification, morphology. Rev. Biol. Trop. 51: 1-287.

Taylor, D. 2004. Revisión Morfológica de caracoles dulceacuícolas, familia Physidae. Comun. Soc. Malacol. Uruguay 8: 279-282.
Varela, M., J. Bechara \& M. Andreani. 1983. Introducción al estudio del bentos del Alto Paraná. Ecosur 10: 103-126.

Walter, H. 1968. Evolution, taxonomic revolution and zoogeographic of the Lymnaeidae. Bull. Am. Malacol. Union: 18-20.

Wilke, T., G. Davis, A. Falniowski, F. Giusti, M. Bodon \& M. Szarowska. 2001. Molecular systematics of Hydrobiidae (Mollusca: Gastropoda: Rissooidea): testing monophyly and phylogenetic relationships. Proc. Acad. Natur. Sci. Philadelphia 151: 1-21.

Zampatti, L. \& G. Darrigran. 2001. Generación y transferencia del conocimiento. El inicio de un intercambio diferente. Fundación CYF, Buenos Aires, Argentina. 
